\title{
Medical Image Analysis: Progress over Two Decades and the Challenges Ahead
}

\author{
James S. Duncan, Senior Member, IEEE, and Nicholas Ayache, Member, IEEE
}

\begin{abstract}
The analysis of medical images has been woven into the fabric of the Pattern Analysis and Machine Intelligence (PAMI) community since the earliest days of these Transactions. Initially, the efforts in this area were seen as applying pattern analysis and computer vision techniques to another interesting dataset. However, over the last two to three decades, the unique nature of the problems presented within this area of study have led to the development of a new discipline in its own right. Examples of these include: the types of image information that are acquired, the fully three-dimensional image data, the nonrigid nature of object motion and deformation, and the statistical variation of both the underlying normal and abnormal ground truth. In this paper, we look at progress in the field over the last 20 years and suggest some of the challenges that remain for the years to come.
\end{abstract}

Index Terms-Medical imaging, medical image analysis, computer vision, image segmentation, image registration, nonrigid motion, deformable contours, image-guided surgery.

\section{INTRODUCTION AND OVERVIEW}

COMPUTER processing and analysis of medical images covers a broad number of potential topic areas, including image acquisition, image formation/reconstruction, image enhancement, image compression and storage, image analysis, and image-based visualization. In this review, we aim to critique the research efforts that have been put forth specifically in the area of medical image analysis over the past 20 years or so. For our purposes here, we will define this area as including the development of the following methodologies: image segmentation, image matching/registration, motion tracking and change detection from image sequences, and the measurement of anatomical and physiological parameters from images. Furthermore, we assume that research efforts related to these methodologies are the key elements of solutions to more systems-oriented problems. Such problems include image-guided surgery/intervention, atlas-based description of entire anatomical regions, deformation analysis based on biomechanical and other models, and visualization of anatomical and physiological processes. With these definitions and assumptions, we have intended to narrow the discussion in this paper to the medical image analysis tasks that are closest to the core mission of the IEEE Transactions on Pattern Analysis and Machine Intelligence.

In this light, we note that medical image analysis has evolved over these last 20 plus years from a variety of directions, ranging from efforts that were natural followons to the development of the image acquisition equipment to those that were motivated from work in the parallel fields

- J.S. Duncan is with the Departments of Diagnostic Radiology and Electrical Engineering, Yale University, 333 Cedar Street, New Haven, CT 06520-8042. E-mail: james.duncan@yale.edu.

- N. Ayache is with INRIA, 2004 route des Lucioles BP 93, 06902-Sophia Antipolis Cedex, France. E-mail: nicholas.ayache@inria.fr.

Manuscript received 19 Aug. 1999; accepted 24 Nov. 1999.

Recommended for acceptance by K. Bowyer.

For information on obtaining reprints of this article, please send e-mail to: tpami@computer.org, and reference IEEECS Log Number 110458. of pattern recognition, image processing, and computer vision. To be more concrete regarding the first direction, take for instance, an example that evolved from efforts related to image acquisition that began 10 years prior to the time frame we are looking at-the analysis of cardiac nuclear medicine images. Initially, as cardiac nuclear medicine images became available, simply viewing the change of the volume of blood in the heart between enddiastole (full expansion) and end-systole (full contraction) was an exciting use of this new modality. However, the facts that 1) the data were acquired in digital form and 2) that it was feasible that a computer could semiautomatically outline a "region of interest" that contained the entire left ventricle, readily facilitated the measurement of a quantitative index of ejection fraction [1], making this one of the earliest forms of "digital image analysis" that became clinically useful.

From the second direction mentioned above, in the prePAMI era of the 1970s, a relatively smaller group of researchers were involved with treating medical and biomedical image analysis as a unique information processing problem, one where approaches based in pattern recognition, image/signal processing, and computer vision may play a role. Examples of this included work to automatically locate tumors using pattern recognitionrelated methods from Sklansky and Ballard [2]. Other efforts, such as the work of Pizer and Todd-Pokropek [3], emphasized image enhancement and display strategies, realizing that these were critical issues to the end-users (radiologists and others). In this light, one community of researchers, based originally in nuclear medicine imaging, appreciated early-on that the mathematics of forming crosssectional images (i.e., image reconstruction from projections) and that of processing and analyzing images had something in common. This community, bonded by a small, high quality workshop begun in 1969 that was originally titled Information Processing in Scintigraphy and later renamed Information Processing in Medical Imaging (IPMI), still carries on this tradition today [4]. 
Most of the efforts reviewed in this paper are more related to this second direction of development, where ideas are motivated from concepts of pattern recognition, image processing, and computer vision. However, with this in mind, it is important to realize that most medical image analysis efforts are heavily influenced, if not fundamentally driven by, the particular image datasets being utilized and the clinical or biological tasks that underlie the need for image analysis. Thus, although we will review work in the field from an analysis-methodology-based viewpoint, we will attempt to point out where the intersections and overlaps are with both the image acquisition/instrumentation and clinical imaging communities where appropriate. We note that companion articles written about medical image analysis written from the viewpoints of these other communities would be interesting additions to the literature, but are felt to be more appropriate for either imaging-modality-based or clinically positioned journals. Furthermore, our focus will be on methods aimed at analyzing organ-level medical images (i.e., we won't say much about the analysis of microscopy-acquired images common in biology). Finally, we emphasize that medical image analysis encompasses the analysis of both anatomy/structure and function, although much of the work in the field has focused on structural analysis. While this paper reflects this bias to some extent, we note that the analysis of physiological function from images is an area of equal or greater importance in the years ahead. In the discussion below, focusing on methodology issues framed in this paragraph, we will divide the last 20 years into four time frames:

1. pre-1980 to 1984 , that one could term the era of $2 \mathrm{D}$ image analysis,

2. 1985-1991, when knowledge-based strategies came to the forefront and the advent of Magnetic Resonance Imaging (MRI) changed the landscape,

3. 1992-1998, when the analysis of fully 3D images became a key goal and more mathematical-modeldriven approaches became computationally feasible, and

4. 1999 and beyond, where now advanced imaging and computing technology is facilitating work in imageguided procedures and more realistic visualizations.

Finally, in the conclusions, we will take stock of the state of the art of the field, including a look at the remaining challenges we face.

Several final introductory points that may be useful to the more general computer vision-oriented reader relate to the unique nature of the medical image datasets that form the basis of this field. In most of computer vision, one is concerned with issues related to 1) the vagaries of variable placement of light sources and, hence, illumination and 2 ) recovering depth. These issues either don't exist or come in markedly different forms in medical image analysis. Here, concepts of "varying illumination" are generally not relevant in exactly the same manner, although, in some cases, for instance due to bias field inhomogeneities in MRI, the image intensity in a homogeneous region of tissue can vary as a function of distance from the "sensor" (i.e., the RF coils). Also, due to the typically tomographic nature of the image data and the ability to acquire full volumes of data in many modalities, recovering depth is often a nonissue. Thus, in general, while certainly many of the problems and issues being addressed in medical image analysis have physics-based components and 3D processing issues that must be carefully considered: 1) It's not typically the case that the physics of optics and light reflection/ absorption that are of concern, but rather the physics of a variety of energy sources interacting with living tissue, and 2) it's not the recovery of depth that is required to segment or measure 3D structure, but rather one must consider how to extract information about shape, motion, etc., from a fully $3 \mathrm{D}$ volumetric dataset.

It may also be helpful to the reader to note that there have been several other articles that have reviewed the medical image analysis field from a variety of perspectives in recent years. References to these are included below, throughout the text, and include general overviews [5], [6], reviews of image segmentation [7], reviews of image registration [8], an overview of clinical applications of virtual reality and surgical simulation (e.g., [9]), and an entire edited book on Computer Integrated Surgery [10].

\section{Pre-1980 to 1984: The Era of Pattern RECOGNITION ANALYSIS OF 2D IMAGES}

During the early years of the IEEE Transactions on Pattern Analysis and Machine Intelligence, the term "medical image analysis" was not yet in common use. However, a variety of meetings had included work related to the analysis of medical and biomedical images. The state of the fledgling field of medical image analysis in the early 1980s can be seen by observing the relevant subject areas covered by a conference on this topic that occurred in this time frame. The proceedings of the IEEE Computer Society's 1982 International Symposium on Medical Imaging and Image Interpretation held in Munich, Germany [11] listed technical sessions dedicated to 1) the image processing topics of enhancement and reconstruction, 2) the development of specialized image processing hardware, and 3) sessions on feature extraction, image segmentation, and structural/ statistical pattern recognition. It is this third group of topics that was most relevant to what is widely thought of today as medical image analysis. Within this group, a variety of applications were addressed at the 1982 meeting, ranging from quantifying structure in cell microscopy to growing regions in $\mathrm{x}$-ray angiograms for finding vessels to finding the outline of the heart in gated blood-pool nuclear medicine images. One forward-looking paper [12] even reported on an early strategy to utilize Computed Tomographic (CT) images as the basis for planning operations in neurosurgery. A particular characteristic of most of the work during these years was that researchers were primarily thinking in terms of analyzing two-dimensional (2D) image datasets.

As another interesting way to observe work performed during this era, one could look at a snapshot of three different key areas of effort aimed at 1) segmenting structures, 2) registering two image datasets, and 3) quantifying cardiac motion from image sequences, all 
problems that continue to be addressed in 1999. Characteristic of segmentation approaches in this time frame were ideas aimed at first detecting contrast edges from twodimensional image datasets and then performing a basic edge grouping or linking operation using some sort of contour search heuristic with smoothness properties embedded in the figure of merit (e.g., [13]). Such approaches took advantage of some general developments in the image processing/computer vision community, such as in [14], and could be seen as a very crude precursor to the variety of deformable boundary finding approaches in development today. In terms of image matching or registration, perhaps the most prominent application area in the early 1980s was digital subtraction angiography (DSA). Here, vessels were imaged before and after a dye injection and then the two sets of images subtracted. Since the data consisted of two sets of images taken at different points in time with the possibility of motion in between, the patient movement had to be compensated for in order to perform a high-quality subtraction. Image-gray-scale-based matching metrics, such as correlation and the magnitude of the intensity differences, were employed to estimate the rigid mapping parameters that would register the images (e.g., [15], [16]). All of these efforts obviously were aimed at matching 2D image datasets. Interestingly, the same basic problems of which metrics to use, intensity-based or otherwise, remain an open issue in 1999, although now focused more on 3D-to-3D image registration. It is of interest to note that an excellent review of work over the past two decades in motion correction for DSA has been recently published [17]. Some of the early efforts on DSA image matching and subtraction rapidly found their way into commercial imaging products and could be considered to be some of the earliest successes of the field of medical image analysis. It is interesting to note that further work in this area, now related to quantifying coronary stenosis from these images, remains an important issue in the last half of the 1990s (e.g., [18]).

With respect to the quantitative characterization of cardiac motion from image sequences, this work primarily made use of images from x-ray ventriculography, cardiac nuclear medicine, or echocardiography during these years. The clinical state-of-the-art at this point in time was to manually outline the endocardial boundary in two image frames chosen from the sequence to represent end-diastole (ED-when the heart is at rest) and end-systole (ES - when the heart is fully contracted). Computer algorithms were then used to overlay digitized forms of these two contours and to find the center of mass of the ventricular area at ED. Finally, by extending rays from this center in all directions and measuring the changes in radial raylength between ED and ES, a crude approximation of regional wall motion could be calculated [19]. Problems with this strategy include the fact that the heart goes through a wave of motion between ED and ES and does not truly contract radially towards a single central point. Also, the motion along a radius does not represent motion of a single material point. Finally, actual cardiac motion is in 3D. While some research attempted to characterize the wave of cardiac motion (e.g., [20]), most of these issues could not be addressed due to limitations in the cardiac image data available in the early 1980s.

Along very different parallel lines, one effort worth noting during these years, based in the medical physics community, was aimed at defining the basic conditions under which human observers could best detect information from medical images. This area of human psychophysics evaluated image quality for task-specific performance. Work went on across the imaging modalities available at this time (general x-ray, CT, DSA) and resulted in a number of papers defining ways to measure observer performance, best exemplified by the work of Metz at the University of Chicago [21], [22], who applied the idea of using receiver operating characteristics (ROC) to the analysis of medical image data. This approach, borrowed from work dating back to World War II on the evaluation of the performance of radar observers, showed that human observers achieved different error rates under a variety of imaging conditions and prior assumptions. It set the stage for much of the computer aided diagnosis (CAD) work described in the next section and the years beyond. Furthermore, as many lament the lack of solid evaluation in medical image analysis research in 1999, some would point to the lack of attention to these early efforts, as well as to the ongoing efforts in image quality assessment, as one key part of the problem. It remains the case today that the work in the image quality area and the efforts that are typically thought of as "medical image analysis" rarely come in touch with each other, except at a small set of technical meetings [23], [24].

Interestingly, as a final comment on these early years in the field of medical image analysis, we note that some researchers were quite ahead of their time in terms of developing ideas that would eventually become useful. The idea of developing a fully 3D edge detector, useful for finding features in volumetric datasets and published by Zucker and Hummel [25] in 1981, is one such example of this. Reprint requests skyrocketed for this at the turn of the $1990 \mathrm{~s}^{1}$ as 3D data became more and more available, whereas few appreciated the impact the original article would have at the time of publication. Another such idea were efforts to try to apply fully 3D deformable object segmentation strategies to stacks of 2D slice images. The PhD thesis work of Schudy, under the guidance of Dana Ballard and published in 1978, uses a 3D model based on Fourier harmonics to guide the recovery of the heart chamber from sets of ultrasound images [26], an approach not too different from many of the deformable surface strategies ultimately proposed (see Section 4). Finally, the current wave of atlas-based segmentation and registration strategies popular in 1999 certainly have some of their roots in the visionary efforts of Bajcsy et al. in 1983 on the use of elastic matching of atlases to image data [27].

\section{1985-1991: KnOWLedge-BASed APPROACHeS INFLUENCE THE FIELD}

The mid-to-late 1980s were a transitional time for the field. To some extent, research in the classic problems of

1. Personal communication with S. Zucker. 
boundary finding, 2D image matching, and ideas related to pattern recognition-driven, computer-aided diagnosis continued along many of the same directions begun earlier in the decade. Some of these efforts helped lay a solid groundwork for work that still goes on today, helping to shape and refine the problem definitions to the point where more focused efforts could be eventually carried out (e.g., [28]). In fact, some of the earliest computer-aided diagnosis (CAD) work in mammography went on during this time frame. Other work in this era also began to point in different directions, in part influenced by the then-exploding field of artificial intelligence. Ideas of incorporating higher level reasoning strategies into recognition systems that were previously driven by more bottom-up image processing and pattern recognition approaches were already being considered in the mainstream image processing, pattern recognition, and computer vision communities. This thinking began to influence efforts in the medical image analysis community, although with somewhat of a time lag. Often, this work borrowed from ideas being considered in the general computer vision field, although the more solid efforts developed medical-task-specific strategies that incorporated domain-specific information into the knowledge models. Also, with respect to parallel developments in medical image acquisition during this time period, Magnetic Resonance Imaging (MRI) was becoming more and more important as a source of image data. More details on developments in some of the key image analysis methodology areas are described below.

\subsection{Computer-Aided Diagnosis (CAD)}

The first attempts at fully automatic computer-aided diagnosis of x-ray mammograms were proposed in or about 1987 (e.g., [29]) and were based on the image quality assessment (i.e., ROC) work developed earlier in the decade. These efforts applied a variety of thresholding and feature-detection operations to digitized mammographic images and then used linear discriminant functions to attempt to automatically classify normal tissue and calcifications. As noted above, this work was seminal in that it formulated this particular feature detection and classification problem in quantitative terms, with the goal of solving it automatically. Benchmarks were developed for false positive and false negative rates that were used to set goals for future efforts. Steady improvements were then made in the decade that followed by a number of research groups and this work continues today. In fact, there is consensus in a 1999 study by the U.S. National Institutes of Health (NIH) that research dedicated to the automated screening of mammographic image data is a key "bridge" testbed between image analysis algorithm development and routine clinical application [30].

\subsection{Image Segmentation}

Issues related to both general and object-specific segmentation continued to be studied during this time frame, with both boundary finding and intensity-based region growing being considered as alternatives. In the first case, more intelligent approaches to assembling image intensity gradients into coherent structures were being considered in a variety of forms. In the popular area of cardiac image analysis, the use of spatially related knowledge about the bounding walls of the left ventricle of the heart were incorporated into models that guided searches through spaces of detected edges from gated nuclear medicine images [31], [32] and, later, from echocardiographic images [33]. It is important to note that during the later part of this time frame, deformable models were discovered and then introduced into the field [34], although these ideas were developed in more earnest in the early to mid-1990s as discussed in Section 4. On the region growing side, original ideas aimed at utilizing the multiparameter data inherent in magnetic resonance (MR) images to segment structure based on actual physical signal properties was also investigated. Here, basic decision tree (minimum spanning tree) [35] and statistical clustering strategies [36] were employed to attempt to separate gray matter from white matter using mathematically constructed images of the three MR imaging parameters, T1, T2, and Proton Density, estimated from multiecho MR acquisitions. An example of these results is shown in Fig. 1. Note that, while some reasonable grouping of intensity data was possible, true 3D segmentation was not yet possible, in part due to the very thick MR slices $(7-10 \mathrm{~mm})$ that represented the state of the art acquisitions required at this point in time to gain acceptable signal-to-noise ratios.

Another interesting notion related to segmentation brought up during this time frame was the concept of applying forms of scale space theory to the problem, addressing the idea that relevant medical image features show up at a variety of scales, even within the same image dataset. Intending to apply the basic theory developed by Koenderinck [37] and others, a variety of researchers, many from the Universities of North Carolina in the United States and Utrecht in The Netherlands [38], [39] vigorously pursued this direction during this time period with the idea being that alternative spaces derived from scale-space hierarchies of intensity extrema would be useful in negotiating the segmentation (automatic or semiautomatic) of complex medical image data. These approaches ultimately lead to the notions of combining medial primitives with scale space concepts to develop a unified approach to shape description, useful for guiding object segmentation. Perhaps some of the most serious initial attempts at developing basic theories for medical image analysis stemmed from this work during this time period and beyond. However, these approaches generally met with limited success during these years and through the early-tomid-1990s. Nonetheless, these research teams persisted and the ideas are starting to show some promise in the late 1990s, as mentioned below (e.g., [40]).

It is also of interest to note that image texture was pursued as a feature useful for grouping and measurement in image analysis during this period (e.g., [41]), especially with respect to ultrasound image data, including for motion analysis of echocardiographic image sequences [42]. Texture continues to be of interest in the mid-to-late 1990s for trying to follow speckle patterns in echocardiograms [43] in order to track motion and as a feature for segmentation and measurement. While a better understanding of the structure of the speckle and scatter in 


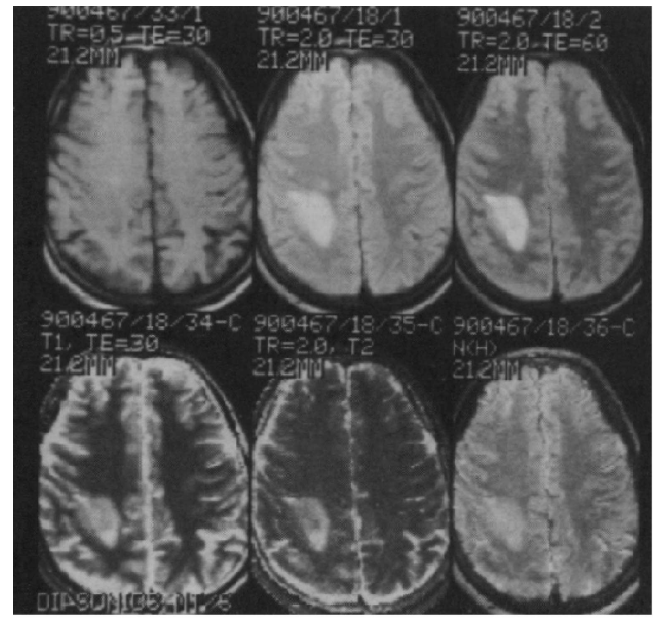

(a)

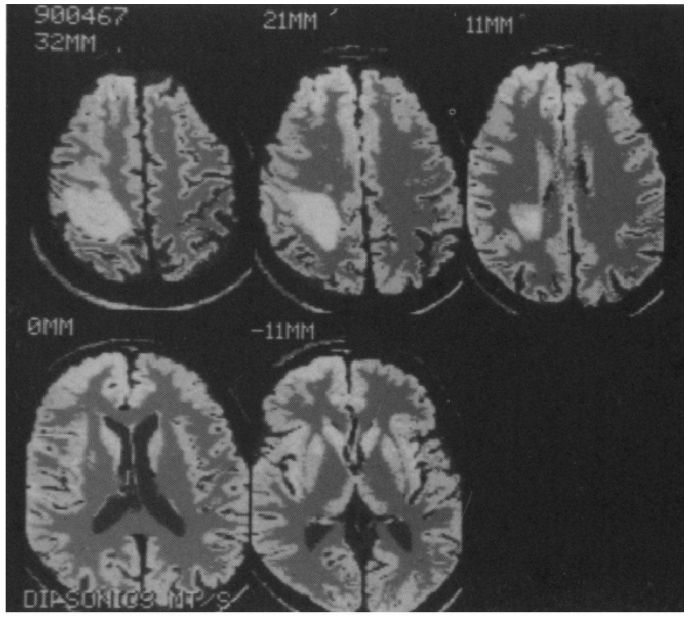

(b)

Fig. 1. Intensity-based segmentation of MR images circa 1987 using cluster analysis of parametric images. (a) Top row: MR basis images acquired at one slice level of a patient with a glioma using $1.0 \mathrm{~cm}$ section thickness, $0.9 \mathrm{~mm} \times 0.9 \mathrm{~mm}$ pixel resolution, TR=.5 s, $2.0 \mathrm{~s}, 2.0 \mathrm{~s}$ (left to right), TE = $30 \mathrm{~ms}, 30 \mathrm{~ms}, 60 \mathrm{~ms}$ (left to right). Bottom row: calculated parametric images (T1, T2, PD) at this same slice level. (b) Segmented regions for same patient in five contiguous image slices (top row, middle image is the same slice level shown in (a)). Segmentation was performed by first using minimum spanning tree-based cluster analysis and then merging the results into four types of tissue: gray matter (light gray), white matter (dark gray), CSF (black), and tumor (light gray/white) [36] (images courtesy of D. Ortendahl, Toshiba).

ultrasound images may be helped by studying image texture, the use of this property has been rather limited to date. However, currently, some of the most promising efforts in extracting boundaries from echocardiographic images employ both temporal boundary tracking and low level, texture-like, feature extraction [44].

\subsection{Image Registration/Matching}

In the late 1980s, clinicians, physicists, and image analysis researchers began to think seriously about integrating different forms of image-derived information. At the forefront of this thinking was the notion that images showing functional information and more spatially detailed anatomical information could be combined or "fused" into a single useful display and analysis platform. The critical problem that had to be overcome to permit this fusion was the fact that two 3D sets of disparate image data, typically acquired at two different points in time and often acquired from disparate imaging modalities, had to be registered/ brought into correspondence. Applications requiring such methodology included surgical planning, radiotherapy, and characterization of difficult-to-analyze neurological disorders. Buried within these problems were questions about what exactly was to be registered. For instance, should one register based on image intensity values or features related to the boundaries of certain anatomical structures? The best known early attempt at the registration of 3D image datasets from two different modalities was an algorithm that matched the bounding surfaces of the brain that were manually segmented from either CT, MRI, Single Photon Emission Computed Tomography (SPECT), or Positron Emission Tomography (PET) [45]. The initial application of this idea was to radiotherapy treatment planning. These multimodality registration problems were especially tricky as the images formed from the various imaging modalities produced different types of information, often at different spatial resolutions. Typically, geometrically related cues provided the only chance for finding common information that could be used as the anchor for image registration. In [45] and as shown in Fig. 2, the authors used a simple Euclidean distance metric to estimate the rigid mapping parameters that will bring one surface in correspondence with the other. As noted above, the surfaces used as the basis for registration were formed from a careful slice-byslice tracing of the outline of the brain. While this worked quite well in many applications, this up-front segmentation was extremely tedious, especially in the case of lowerspatial-resolution datasets such as SPECT and PET.

Alternative image registration strategies, such as registering point clouds of features (such as in [46]) or efficiently matching intensity-based information, had not been developed in this time frame. While basic brute force correlation of image intensity information was always an option for matching 2D image datasets (as noted earlier for DSA), this was computationally impractical for registering pairs of 3D images during these years.

\subsection{Motion Analysis}

The analysis of information embedded in temporal sequences of images was taking hold more and more as a research activity, with the primary applications coming in the cardiac and cardiovascular clinical areas. Image analysis researchers began to better appreciate that the cardiac wall actually goes through a wave of motion from end-diastole to end-systole and that tracking material points on the LV wall was important to characterize meaningful functional changes. Thus, efforts were begun to develop techniques that could analyze this nonrigid motion more completely. Some efforts used the knowledge-based strategies popular in the computer vision community during this time period, trying to develop descriptive models of the shape and motion of the LV chamber. For instance, in the early-to-mid 


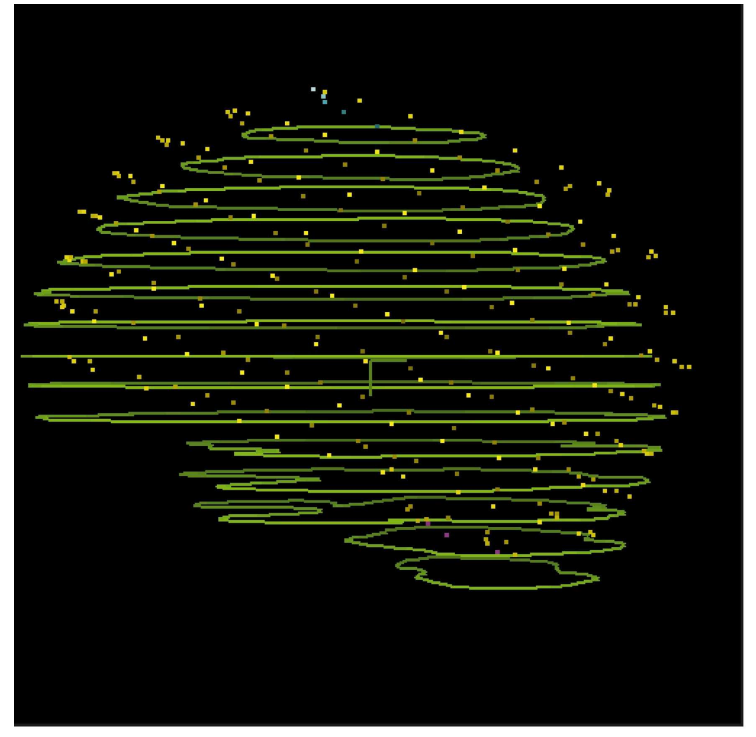

(a)

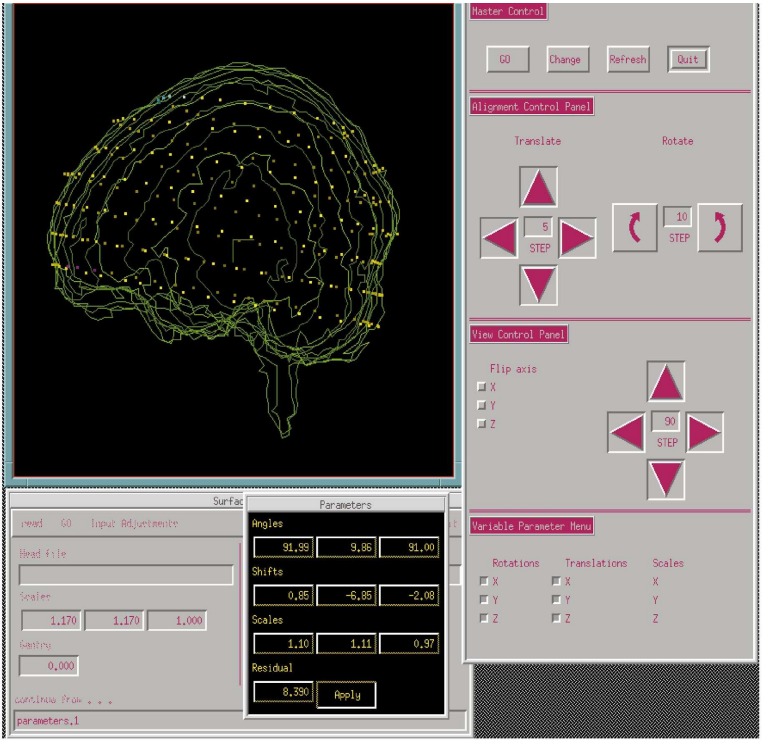

(b)

Fig. 2. Surface-Based Registration of a MRI dataset with a PET dataset using the method of [45]. (a) The contour lines show tracings of the brain surface from MRI and the dot patterns show the part of the surface identified from PET data. (b) Shown here is the control panel used to interactively initialize and/or correct the registration algorithm (images courtesy of C. Pelizzari, University of Chicago).

1980s, Tsotsos [47] and colleagues developed an approach that incorporated higher order motion information derived from radiographic images that described how the left ventricle of the heart moves. This information was assembled in a semantic net and was integrated with lower level image processing and feature detection to create a more intelligent "knowledge-based" expert system model for recovering heart wall motion information. This work was followed by efforts of other groups to incorporate knowledge for finding heart wall boundaries and quantifying motion from nuclear medicine gated blood pool images [31], [32]. One important concept missing from these efforts was the notion of tracking specific material points on the heart wall. However, by the early 1990s, a number of groups began to develop techniques to find correspondences in pairs of frames from 2D cardiac image sequences [48] as well as to parametrize the full spatiotemporal dataset in attempts to model and more fully determine nonrigid motion [49], [50]. In fact, all of these efforts were initially reported at the first conference session on nonrigid motion analysis at the IEEE Computer Society's 1991 Conference on Computer Vision and Pattern Recognition (CVPR91). We note as an aside that the notion that problems encountered in the medical image analysis area can motivate the development of a technical session at CVPR in this manner is important. Such cross-fertilization is needed to encourage medical image analysis researchers to continue to be involved with the PAMI community.

\section{1992-1998: 3D IMAGes AND Toward MoRE INTEGRATED ANALYSIS}

The types of problems addressed in the early part of the 1990s were heavily influenced by the explosion of useful image data now accessible, in a large part due to the increased availability of high quality Magnetic Resonance images from both research laboratories and clinical facilities. Full 3D MRI datasets with better and better soft tissue definition and higher spatial resolution in all three spatial dimensions were becoming available from these imagers, which themselves were becoming available to more and more institutions. Other medical imaging modalities improved considerably during this time frame as well and included the advent of fast spiral-CT, full 3D ultrasound, and the more routine use of 3D SPECT and PET. Driven by the ongoing needs of clinical colleagues, as well as research colleagues in areas such as the neurosciences and the cardiovascular sciences, medical image analysis now required new levels of sophistication to handle more and more complex problems exposed by rapidly maturing data types and greater data fidelity. For instance, the advent of MR tagging and phase contrast velocity imaging now required new approaches for assembling data related to cardiac deformation in three dimensions over time (e.g., [51]). Also, higher 3D MRI spatial resolution facilitated better visualization and also more accurate segmentation and measurement of neuroanatomical structure in the brain. These became important issues for many neuroscience investigators interested in recognizing and quantifying structure related to a variety of neurological disorders (e.g., [52]). Furthermore, as a continuation of ideas forming in the later part of the 1980s, it was realized in the early part of this decade that there would be a significant need for improved methodology development in three key areas across many applications, with the efforts now focused on handling information from 3D image datasets. These areas were: 1) integrating functional and anatomical information from a variety of sources (e.g., MRI and SPECT/PET) (e.g., [53]), 2) pooling data within patient groups (e.g., [54]), and 3) comparing information across different subject populations (e.g., [55]). Many of these problems required segmenting subtle structure when feature information was 
incomplete and registering two 3D datasets when some of the key features were difficult to see: in a nutshell, solving a variety of ill-posed problems. Because of this, the need to incorporate more and more context-based, constraining information within robust forms of decision making was fast becoming one of the key information processing problems to be solved withing medical imaging and medical image analysis.

In a related manner, it became clear that, methodologywise, the desire to integrate different abstraction levels of knowledge into a variety of medical image analysis problems became more pronounced in the mid-1990s. This was in part due to a new appreciation that algorithm development must go hand in hand with some form of validation and evaluation testing on a range of human image data. This, in turn, drove home the idea that robustness over the variability inherent in image data (even from the same modality), the patient population, and algorithm initialization conditions was now critical. Although certainly not ubiquitous in all parts of the medical image analysis literature, there was a sense that the core approaches that underpinned and drove much of the key work in this time frame were beginning to move out of the realm of using expert system-like approaches and more into the world of mathematical optimization, particularly toward nonlinear optimization. Some key examples of this are noted below in Section 4.1.

Before discussing these examples, it is interesting to note that there were two watershed points between 1992 and 1998 that helped mold the field. First, a smallish workshop was organized under the auspices of the American Association for Artificial Intelligence (AAAI) and held at Stanford University in the Spring of 1994 [56]. For the first time, researchers who had long worked on a variety of medical image processing and analysis problems out of traditions with strong ties to clinical collaborators, came together with researchers coming from the "mainstream" computer vision and pattern recognition communities who had now become interested in medical imaging problems. While there is no directly traceable path to this workshop, discussions at this meeting began to mature the field in many ways. The second watershed point was the merging of three conferences (Computer Vision, Visualization, and Robotics in Medicine (CVRMed), Visualization in Biomedical Computing (VBC), and Medical Robotics and ComputerAssisted Surgery (MRCAS)) into a single flagship meeting for the fields of medical image analysis, biomedical visualization, and computer-assisted intervention, now known as Medical Image Computing and Computer Assisted Intervention (MICCAI). This combined meeting was held for the first time at the Massachusetts Institute of Technology (MIT) in the fall of 1998 [57]. In addition to these two watershed events, the strengthening of the IEEE Transactions on Medical Imaging with appointments of more associate editors in image analysis areas and the formation of a journal focused directly on the field, (Medical Image Analysis, Elsevier) helped coalesce researchers and efforts in this area in the middle of the 1990s.

\subsection{Methodology Development: Optimization-Based Decision Making and Information Integration Dominate}

\subsubsection{Image Segmentation}

Probably the most visible approaches brought to maturation during this time frame in terms of both methodology development and application were boundary finding strategies based on deformable models. These methods were now coming into their own for medical applications, as different groups developed fully 3D "snakes" (e.g., the "balloons" approach of [58]) that could be run on volumetric image datasets. In Addition, approaches that incorporated shape priors were also extended into 3D (e.g., [59]), and another line of research pursued by Cootes et al. reported novel ways of introducing priors using point sets that showed promise [60]. Szekély et al. later combined these two ideas to develop a robust 3D strategy [61]. Other interesting approaches included making use of gradient vectors (instead of just gradient magnitude) to guide the contour/surface deformation [62]. Efforts that use deformable models, up until 1996, are nicely summarized in a review article by McInerney and Terzopoulos [7]. While deformable models and active contour-based segmentation have often been claimed as one of the key successes of the computer vision community in the past several decades, one of the most fertile grounds for further development and application of these ideas certainly has been within the medical image analysis community. It is of interest to note that basically all of these strategies were implemented by optimizing objective functions of one form or another, with the most basic forms simply trying to find a compromise between some image-based energy term and another term related to an internal energy or shape model (typically, just smoothness of adjacent points).

An interesting alternative to objective function-based deformable contours also emerged in this time frame. These efforts posed contour deformation as a propagating wavefront that can be seen as the zero level set of an evolving function. This evolving function can then be further captured in the form of a partial differential equation where a speed term forces the propagation to stop according to image-derived information (e.g., edges). The efforts in this area has its roots in the work of Osher and Sethian [63] and was brought into the medical arena by a variety of researchers [64], [65], [66]. Others have taken this idea even further by now coupling evolving level sets to look for the outer cortex of the brain [67], [65], with one version using the spatial constraint that the cortical gray matter is approximately of equal thickness over much of the brain surface [67]. Results using this approach are shown in Fig. 3. It may be interesting to compare the efforts in the early 1980s aimed at separating gray and white matter (everywhere in the brain) using intensity clustering techniques, one result of which was shown in Fig. 1, to this approach. While these earlier approaches used spatially unconstrained region growing methods to cluster similar intensity values, the approach shown in Fig. 3 uses seeds of the bias field-corrected T1-weighted gray level values to help find homogeneous values in between the two (coupled) evolving surfaces, but now also uses spatial 

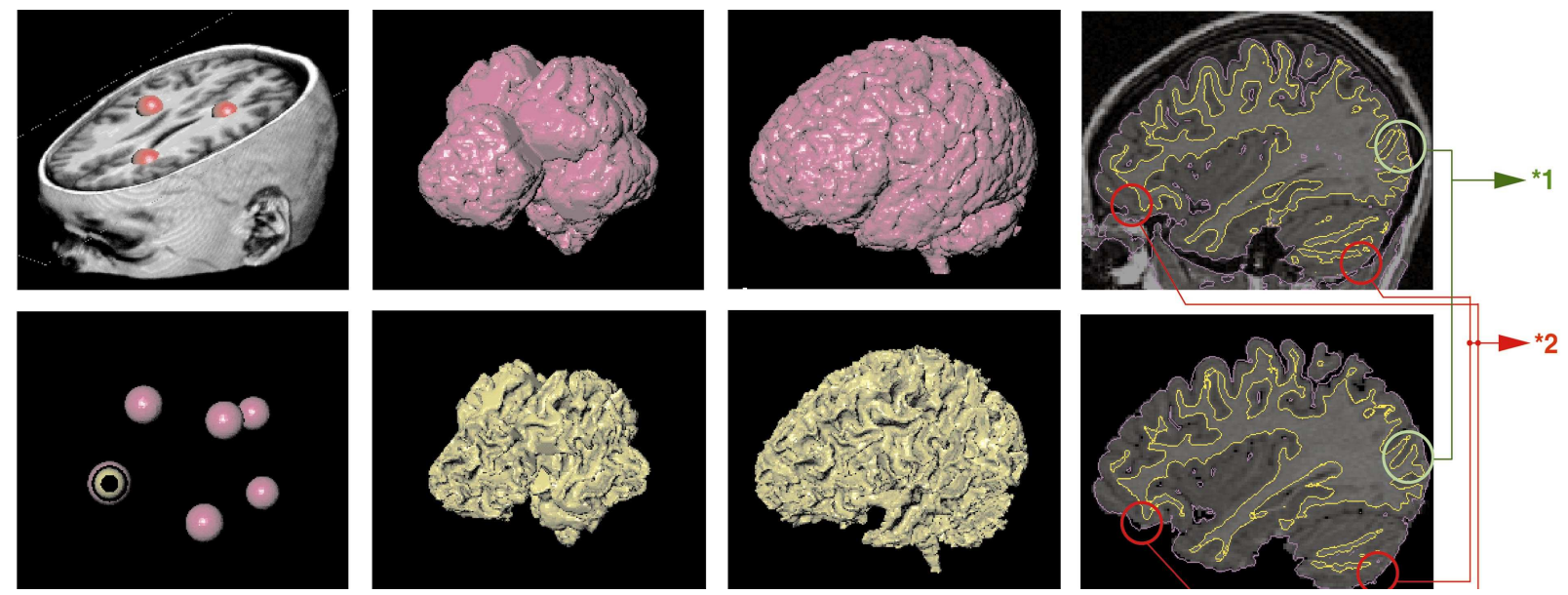

Fig. 3. Results of cortical gray matter segmentation using coupled level sets [67]. (a) Initialization of pairs of concentric spheres in 3D MR brain images; (b) intermediate step; (c) final result of the outer (top) and inner (bottom) cortical surfaces of the frontal lobe; (d) single vs. coupled surfaces approach. Upper: surfaces resulting from finding the inner and outer cortex separately, shown on a sagittal slice through the 3D result; lower: results from the coupled surfaces approach run on original 3D data overlayed on a sagittal slice of the expert tracing result. The outer cortical surface resulting from the coupled algorithm nicely fits the boundary from the expert tracing. Coupling prevents the inner surface from collapsing into CSF (*1) and the outer surface from penetrating nonbrain tissue ( $\left.{ }^{\star} 2\right)$. (Images courtesy of X. Zeng and J. Duncan, Yale University).

constraints to guide the segmentation process as well. We also note that, recently, one group has been able to adapt the level set approach to extract tubular structure from medical images by utilizing notions of codimensionality [68]. A key application here is the segmentation of vasculature.

Despite the successes noted above, it is fair to say that, as the 1990s draw to a close, no one algorithm can robustly segment a variety of relevant structure in medical images over a range of datasets. Surface/boundary finding algorithms often remain sensitive to variation in image acquisition parameters and their own initial starting positions. Thus, research is ongoing in this area and there are some very interesting lines of thinking and effort being pursued in addition to the above-mentioned strategies. These include the maturing efforts of looking for scale spacebased medial representations, such as the "cores" work being performed at the University of North Carolina [40] and efforts aimed at integrating boundary-based and intensity-grouping-based strategies to gain robustness to noise, blur, and initialization (e.g., [69], [70]). Another related idea in this category is the integration of gray-scaleintensity-based "appearance" models with the point/surface-based models of image structure that were discussed earlier. The application of these to atlas matching was recently described by Cootes et al. in [71].

It is important to note that intensity-based segmentation of medical image information also matured during these years in some interesting ways. Perhaps most notable is an idea aimed at simultaneously trying to cluster intensity values and correct for image distortions in MR data. The grouping or clustering of intensities was previously always subject to the spatial variation of the signal information within a study or across studies. While somewhat true when using projection X-ray and/or Computed Tomographic (CT) data, this was most true in the everburgeoning world of magnetic resonance imaging. Here, intensity variation due to bias field effects could greatly affect region growing algorithms. A creative idea to simultaneously estimate the bias field and classify intensity regions in the brain was proposed by Wells and colleagues which provided a promising and interesting solution to this problem [72]. The strategy utilized an Expectation-Maximization (EM) approach to go back and forth between the classifying of a bias field-corrected image and estimating the bias field itself. Certainly not all agreed that this was the best strategy, and a number of groups have separately addressed this bias field estimation problem (e.g., [73]). It is interesting to note that work still continues aimed at trying to improve basic intensity-based, region growing strategies for finding bias field inhomogeneities [74] using fuzzy c-means and other approaches. In addition, the problems being addressed (e.g., gray/white matter segmentation) are very similar to those that were worked on back in the mid-1980s. Thus, while image data fidelity has improved greatly over 10 or 12 years, approaches to intensity-based region growing have matured only somewhat during this time period.

Finally, we note that, while some initial efforts have been made in the community to form standardized datasets to evaluate and validate segmentation algorithms on simulated data [75], very little effort has been put forth to organize databases with some form of ground truth, human experts or otherwise, that would permit the community to quantitatively compare and contrast ideas. At least one initial start in this direction is the Internet Brain Segmentation Repository (IBSR) at Harvard which provides manually guided expert segmentation results along with magnetic resonance brain image data. Its purpose is to encourage the evaluation and development of segmentation methods and is based in part on the method described in [76].

\subsubsection{Image Registration}

Image registration continued to be an important and challenging area of research, focusing primarily on the rigid matching of two $3 \mathrm{D}$ image datasets, but with researchers beginning to address the need for nonrigid 
mappings. Approaches are generally separated into those that were feature/surface-based, where some form of sparse spatial information had to be identified or segmented prior to the registration process, or those that were voxel intensity-based, where registrations could be performed right from the gray scale data itself, now a possibility due to the improved computational power now available in this time frame. A more detailed categorization of registration approaches and an excellent review of the entire range of rigid and nonrigid image registration strategies through 1998 is provided in a review article by Maintz and Viergever [8].

As noted above, some of the earliest forms of rigid 3D image registration algorithms relied on minimizing the average Euclidean distance between two (manually) segmented surfaces [45]. This basic approach was later adapted to utilize chamfer distance matching [77] and is included in current commercially available software such as ANALYZER [78]. A number of powerful, alternative surface/ feature-based strategies were now being developed as well. For sets of delineated points, many saw the Besl and Mackay iterative closest point (ICP) approach as an attractive idea [46]. However, this strategy could be quite sensitive to changes in feature locations and false positives in the detection of closest points. This problem was partially fixed in the adaptive-threshold work of Feldmar and Ayache [79]. In addition, other investigators noted the problems with robustness to outliers and proposed an alternative scheme based on assignment matrices and robust statistics [80] known as robust point matching (RPM). As surface segmentation strategies were becoming more reliable, the development of feature-based strategies, where both correspondence and mapping were simultaneously estimated as part of the algorithm, became feasible, as exemplified by the work of Thirion [81] and Guéziec et al. [82] that focused on the extraction and matching of crest lines initially between skull surfaces segmented from human MRI data. An example of this approach is shown in Fig. 7. The ICP and RPM strategies discussed above also implicitly simultaneously solve the correspondence and matching problems. Many groups felt that using these more geometrically based strategies that typically relied on some form of a priori segmentation or point identification would provide the highest degree of registration precision for a variety of problems.

Work on intensity-based rigid image registration also gained much attention at the midpoint of this decade, first through efforts from within the neuroscience community to develop an approach that matched 3D image datasets by optimizing a metric related to the ratios of local intensity variances in the two image datasets [83]. Later, more analytically trackable efforts on intensity-based matching emerged simultaneously in the mid-1990s from groups at MIT [84] and Leuven, Belgium [85], that employed information-theoretic metrics to match intensity values in two sets of images. These mutual information strategies proved to be quite robust across a host of 3D image registration problems and have been successfully applied to a variety of problems, including intermodality registration of functional and structural information, as seen in Fig. 4, and in the registration of ultrasound images acquired at different time points, as shown nicely for a breast imaging application in Fig. 5 [86]. In addition, during this time frame, the evaluation of registration approaches finally received some serious attention. A study was performed by West and Fitzpatrick at Vanderbilt University to directly compare image registration algorithms aimed at estimating rigid mappings on a brain dataset with known ground truth [87]. While the results were not definitive with respect to the overall strength of any one algorithm, they did reinforce the idea that intensity-based metrics were quite competitive. This has stimulated further work along these lines, including recent efforts to develop new intensity-based registration metrics [88].

Many, if not most, of the application areas that incorporate image registration ultimately require nonrigid mapping schemes. This is especially true when trying to relate information between two sets of images from two different patients or between an atlas and a particular patient's images. To do this, of course, a nonlinear parametrization of the mapping that is being estimated must be chosen. While many groups began with trying to estimate affine mappings, hoping to move later to fully nonlinear schemes, some realized the importance of trying to obtain proper nonlinear interpolants from the start. Some of the most prominent work here was that of Bookstein [90], [91], who argued strongly that thin-plate splines were the appropriate choice based on the statistics of morphology. Other efforts felt that models from fluid mechanics [92] provided the most suitable mechanisms for estimating nonlinear mappings for a range of intra- or interpatient registration problems. While mathematically quite interesting and rather robust, some would say that adhering too closely to the notion that these mappings represent the real physics of these problems (i.e., that one brain can be accurately physically deformed into another according to the laws of fluid mechanics) is problematic. Nonetheless, these approaches are capable of producing interesting and usable results. This is illustrated nicely in Fig. 6, where an iterative volumetric deformation (using the algorithm described in [93]) is applied to a 3D MRI image that is part of a deformable atlas. As shown, this volume is deformed to match the 3D target volume of a particular patient's MRI study. Shown on the top part of the figure is a view of the skin surface as the volume deforms. The images in the middle and bottom rows of Fig. 6 show the midsagittal plane of the template, target, and deformed template for two slices. In both cases, the final transformation was achieved by first elastically deforming the template to get the global shape and then deforming it fluidly to get the local shape.

\subsubsection{Analysis of Structure/Morphology}

The accurate and meaningful measurement of normal and abnormal structure reached new levels in the last half of the 1990s due to both improvements in the underlying image data and the maturation of the image analysis methodologies employed. One aspect of this was that structural measurement was finally moving beyond primarily quantifying volumes to now including shape. 


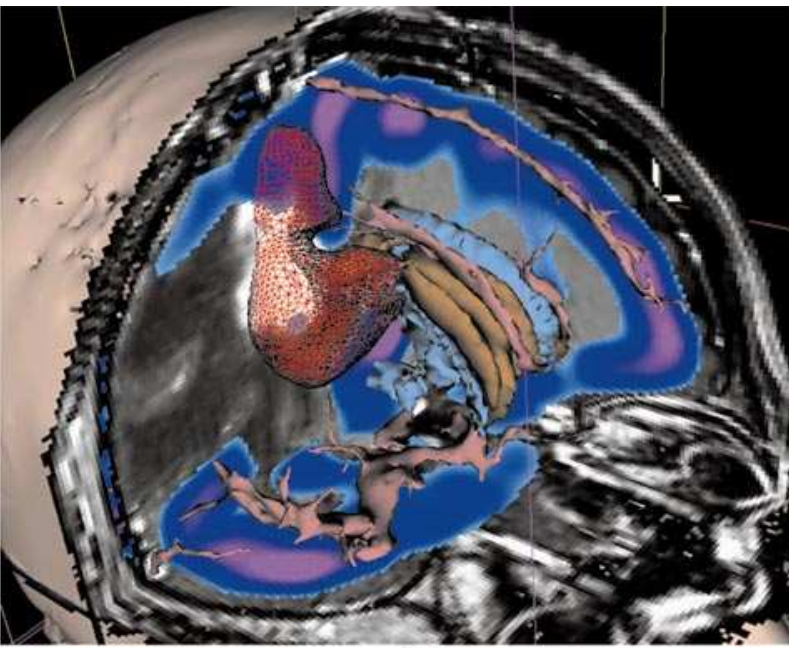

(a)

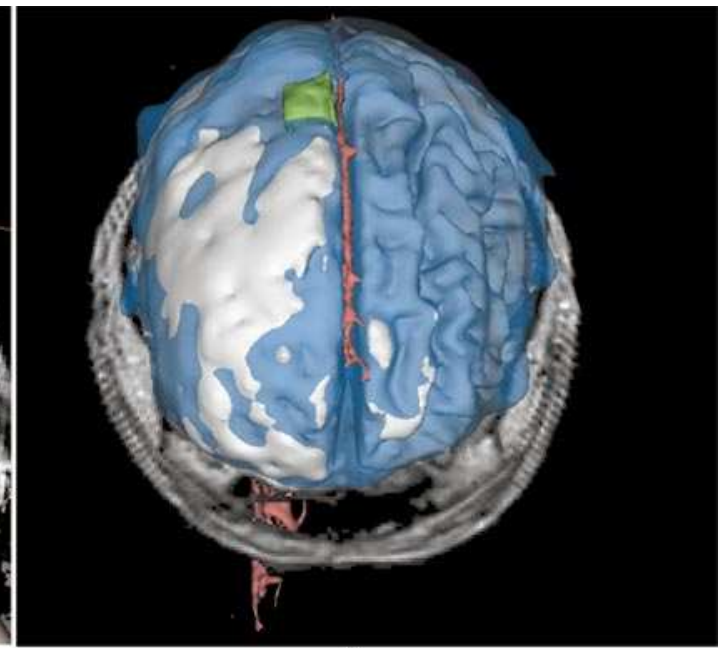

(b)

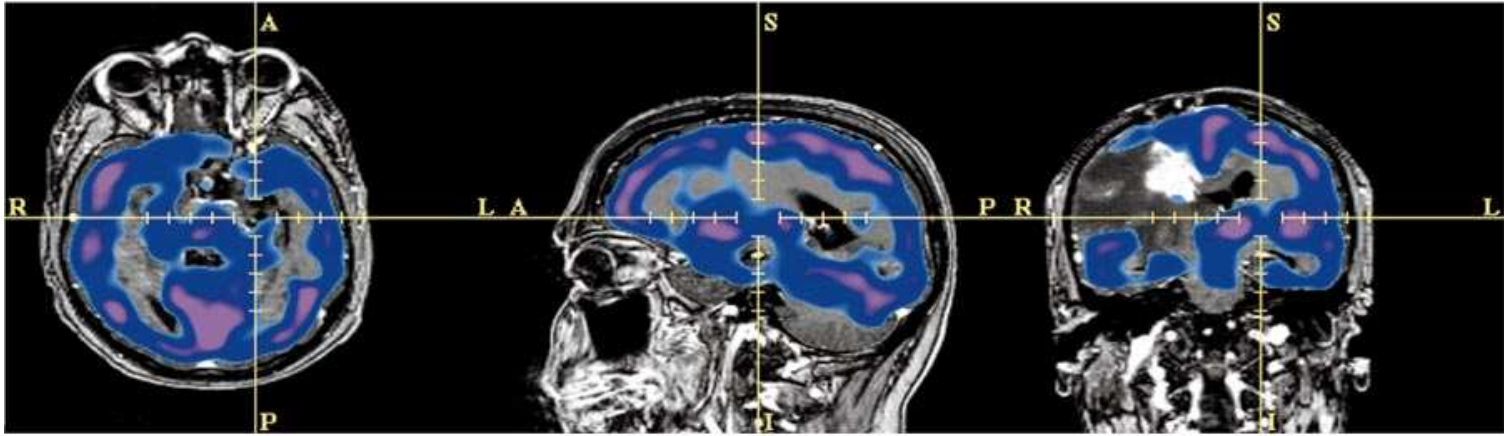

(c)

Fig. 4. Intensity-based registration of an MRI and an integrated thallium/technetium SPECT dataset using mutual information as described in [84]. (a) Shown on top left is a postregistation visualization of key information. The technetium-based data is in blue, the red mesh is from the thallium data, and the underlying gray scale information is from MRI. Note the consistency of the information from the thallium dataset with the bright tumor position as seen in MRI. (b) A visualization of the brain surface from the same MRI dataset (in white) along with an isosurface of the technetium scan displayed as a transparent blue surface model. The lack of perfusion in the vicinity of the (green) tumor due to brain edema is well visualized by the void in the technetium surface. (c) The bottom three images show overlaid orthogonal cuts through the the two registered volumes. (Images courtesy of A. Nabavi and W. Wells, Brigham and Women's Hospital, Harvard University).

It is important to note that these analysis methods require processing beyond the initial steps of segmentation and/or registration of datasets. The study of morphometry, using landmarks of know correspondence and statistical analysis has long been supported by Bookstein, who has found meaningful shape differences in several domains, including schizophrenia [55]. Examples of this include the characterization of structural differences in the brain via the quantification of sulcal depth, cortical thickness and/or cortical shape, (e.g., [94], [67]). Here, an initial segmentation

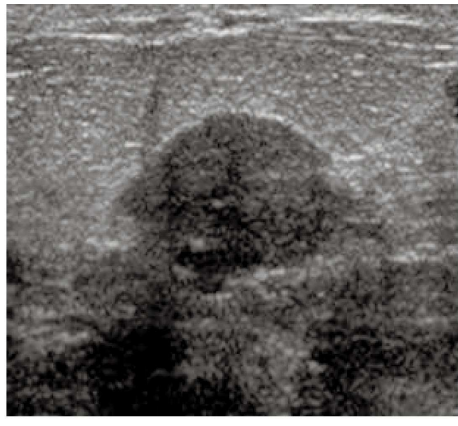

(a)

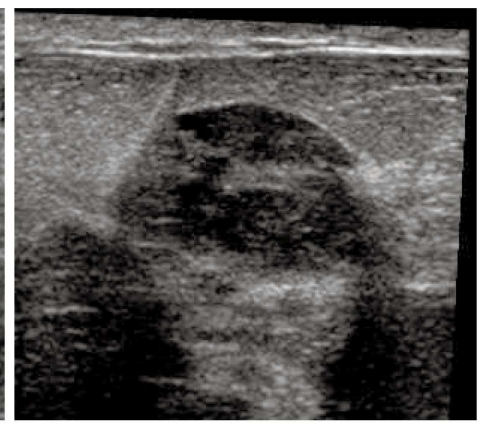

(b)

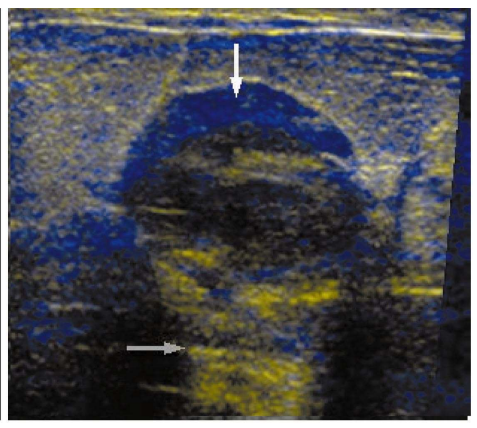

(c)

Fig. 5. Rigid coregistration using the mutual information approach described in [89] of two sequential ultrasound scans of a breast lesion in a patient undergoing chemotherapy. (a) Central B-scan frame of the first scan, obtained one week before the start of therapy, coregistered and transformed to map onto the second scan. (b) Corresponding frame of the second scan, obtained five weeks later. (c) Pseudocolor image of the differences between the two scans, showing increased echogenicity (blue) where the mass shrank (white arrow) and decreased echogenicity (yellow) below the mass (gray arrow). (Images courtesy of C. Meyer, University of Michigan). 


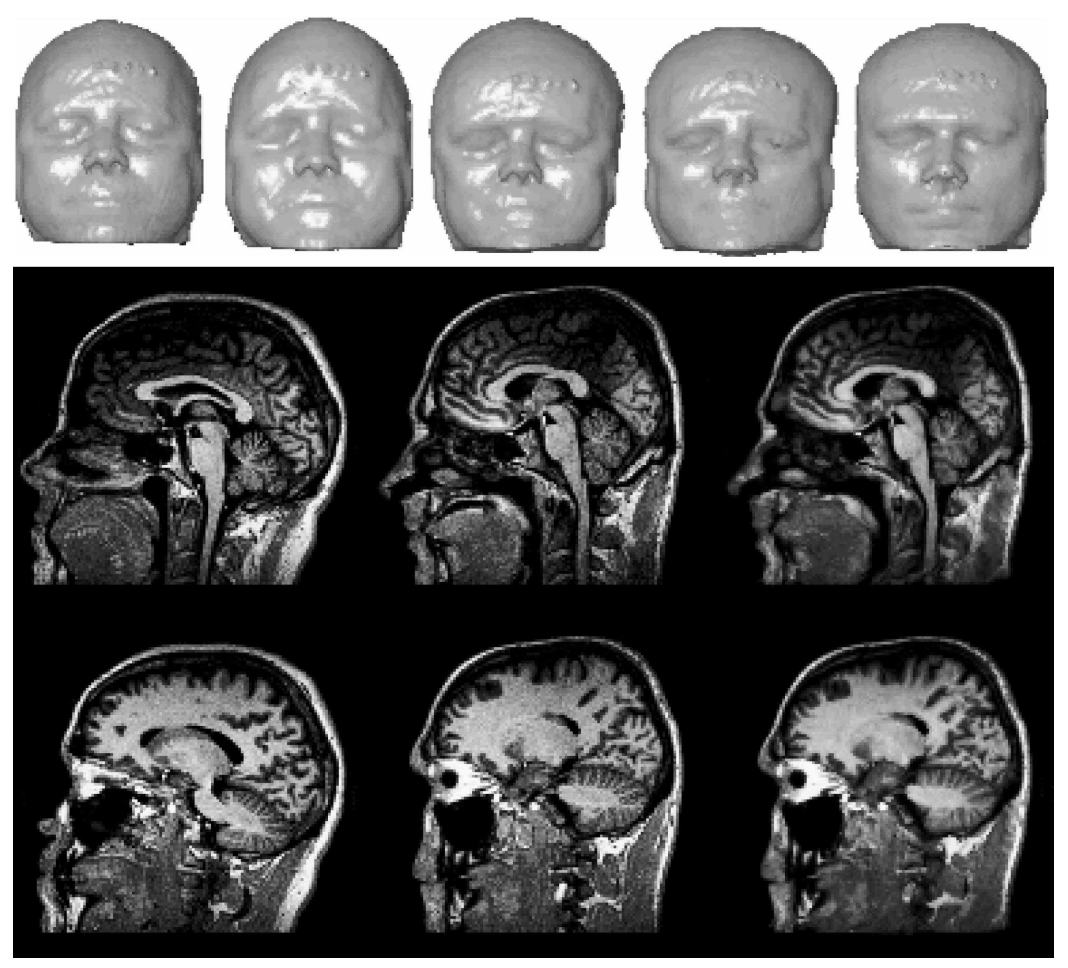

Fig. 6. Top: Three-dimensional facial surface views reconstructed from MRI data of the deformable atlas anatomy as it is automatically deformed into the shape of the study anatomy using a fluid deformation model as described in [93]. The undeformed atlas is shown in the leftmost panel and the final deformed state is shown in the rightmost panel. Middle/Bottom: The middle and bottom rows show two sagittal MRI slices from the MRI atlas (left), study (middle), and fluidly deformed atlas (right), respectively. (Images couresty of G. Christensen, University of lowa).

of the cortical surface(s) is typically performed and then algorithms are run on the resulting segmented information. For instance, in the work of Valliant and Davatzikos [94], special "ribbon" operators were developed to chararacterize the depth of the sulcal grooves. A related example is the automated extraction of sulcal and gyral curves found using by developing crest lines from the differential geometric properties of a segmented brain surface, as shown in Fig. 7. It is important to note that the extraction of shape features from a segmented anatomical object in this way may be useful for not only measurement but for feature-based registration as well [81], [82]. While the brain is an anatomical structure rich in morphological information that can be derived from an accurate segmentation of a particular image, local and global measures of shape may be useful in characterizing many organs and other soft tissue structures, including the heart at rest, the liver, the cartilage in the knee, etc. Furthermore, analysis of bony structure was ongoing during this time frame. One interesting idea already in use in a clinical setting employed composites of $\mathrm{x}$-ray images and semiautomated analysis to measure deformity in the human spine related to scoliosis [95]. The measurement of the articulated motion of bony structure in the movement of the hands, feet, and jaw (for dentistry) are other areas that were under investigation.

\subsubsection{Analysis of Function, Including Motion and Deformation}

The quantification of physiological processes is an important area of growth within the medical image analysis community that began to receive more attention in this time frame. This was due in part to new developments in the functional brain imaging domain, where functional Magnetic Resonance Imaging (fMRI) was now becoming more and more available to neuroscience investigators, adding to the nuclear medicine modalities of SPECT and PET that were already available for studying brain function. One key problem, mentioned earlier, was the need to register any and all of this functional information to higher-resolution anatomical studies. In the fMRI area, the extraction of the underlying information now also depended on statstical decision making. Much of this work had been done by MR physics-based and neuroscience-based investigators, but now medical image analysis researchers were beginning to get involved in these problems (e.g., [96], [97]).

As noted throughout this paper, one area of functional analysis that has clearly been an ongoing research issue in the medical image analysis community is the study of left ventricular (LV) motion and deformation, critical for understanding cardiac function. Again, the ongoing development of new image acquisition technology affected this area during the 1990s. Perhaps most prominent was the develoment of MR tagging [98], [99] that allows for a large number of material points to be marked and tracked over portions of the cardiac cycle in a noninvasive manner. By locally perturbing the magnetization in tissue, one can create spatially encoded patterns such as grids (e.g., [99]). These patterns or magnetization tags are seen as dark regions in subsequent images (within a certain relaxation time $T_{1}$ ). As magnetization moves with tissue, the tags will move in the corresponding images, directly reflecting the motion of the underlying tissue and allowing one to follow 


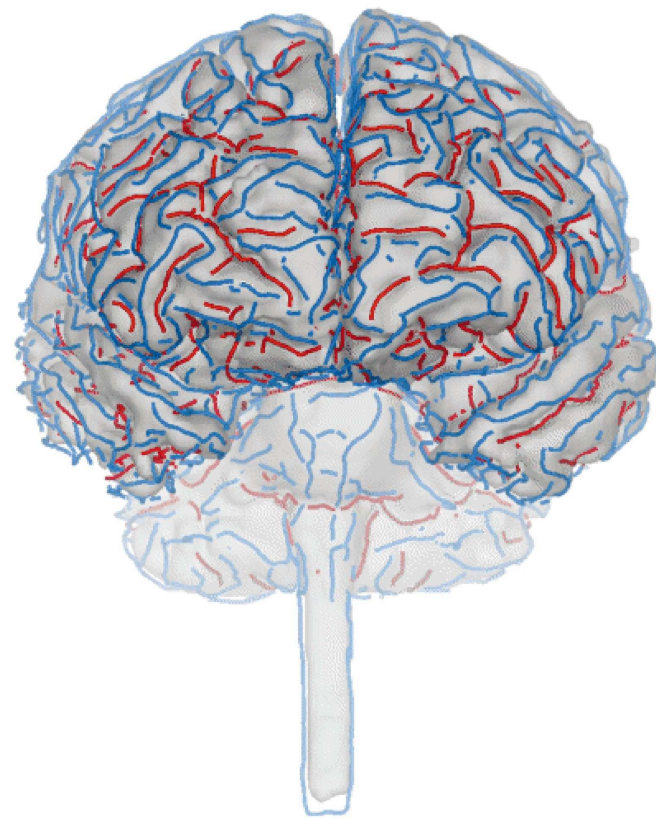

(a)

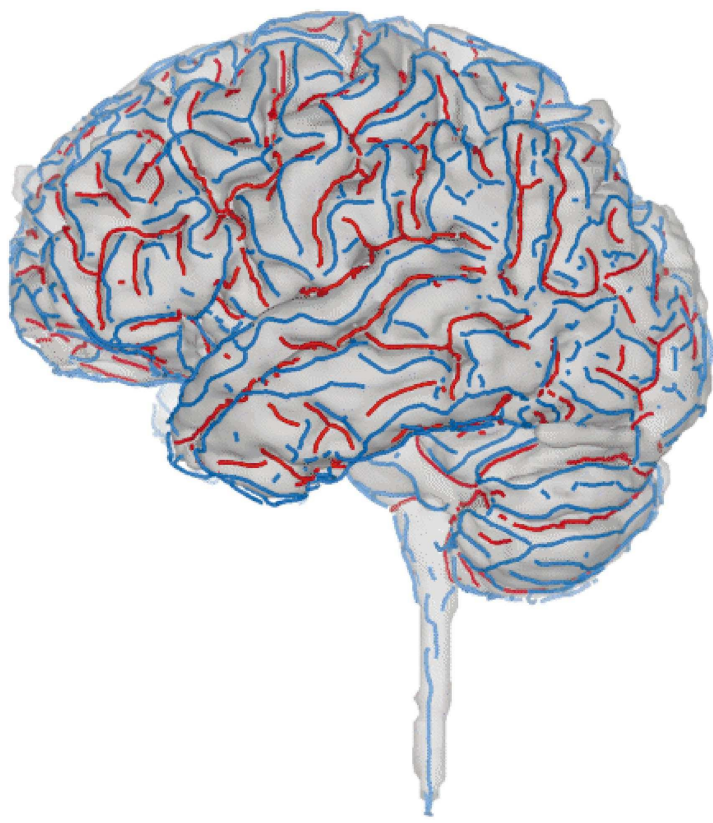

(b)

Fig. 7. Automatic extraction of sulcal and gyral crest lines from a segmented surface of the brain using the method of [81] (figure courtesy of N. Ayache and G. Subsol, INRIA).

the motion patterns within otherwise featureless structures such as the myocardium in the heart wall. Examples of these MR tag images are shown in Fig. 8. While reproducible and accurate detection of these lines in any given image slice is challenging, once the lines are located, a set of grid intersection points can be assembled in $3 \mathrm{D}$ over several periodic time sequences that can be used to compute deformation in the form of spatiallydistributed strains. Procedures for extracting shape and deformation information from MR tag data included efforts using thin plate splines [100], [101] and forms of mechanics-based finite element models. Shown in Fig. 9 are the efforts of Park et al. [51] using volumetric deformable models based on incorporating concepts from Lagrangian mechanics and finite elements. This model was especially interesting for clinical applications as it was able to recover the myocardial twisting motion of the left ventricle (note endocardium in white in Fig. 9). The combination of computed forces from the tag data points from two orthogonal planes allows recovery of the deformation of the model in 3D, ultimately permitting strain measurements to be recorded. This sort of quantitative information about cardiac deformation was quite rich compared to the early forms of measuring LV motion that were used in the early 1980s (see Section 2) to quantify cardiac function that relied on tracings of the endocardial boundary from 2D images at ED and ES. We also note that some interesting work moving toward developing dense strain information in close to real time from MR tag acquisitions was (and continues to be) pursued by Gupta and Prince [102].

MR tagging was not an end-all to cardiac deformation analysis, however, as the tags tended to fade over the cardiac cycle, as shown in Fig. 8. Several alternative approaches to quantitatively recovering the deformation of the left ventricle of the heart were also brought forth during this time period. One of these involved another interesting new MR acquisition sequence known as phase velocity imaging [103]. Here, using velocity encoding gradients, one can force the phase of the MR signal to be directly proportional to a specific range of velocities. The technique can be used to develop dense maps of instantaneous velocities. Both the MR community [104] and the image analysis community have looked into constructing deformation/strain maps from these data, the latter using computational models derived from computer vision work originally aimed at recovering object boundary-constrained optical flow [105]. Another alternative was to follow the shape properties of the bounding walls of the myocardium that could be segmented from each frame. Some groups utilized shape to characterize epicardial motion [106] and others used differential geometric features as a basis to recover endocardial motion [107], [108]. Potentially, these ideas now made the derivation of LV strain measurements possible from any one of a number of imaging modalities, including cine-MRI, cine-CT, and 3D echocardiography. This work is ongoing and some more robust ways of following shape that were proposed in this time frame continue to be explored [109].

We note that the analysis of motion in temporally acquired datasets may also include the characterization of changes found from longitudinal imaging of a patient over periods of days, weeks, or months. This form of analysis can characterize tumor growth, among other things. Often, cancerous lesions are the only object in a medical image that can grow over time in adults. Work on looking for changes in time series data to better understand the multiple sclerosis disease process was carried out by Gerig et al. [110], who developed intensity- 


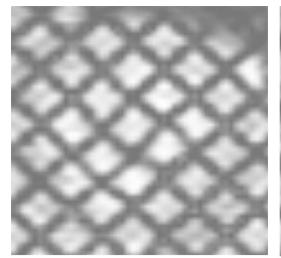

(a)

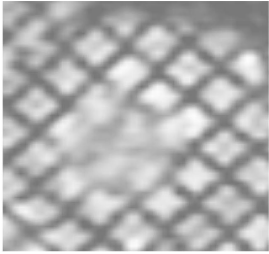

(b)

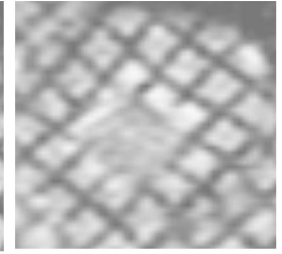

(c)

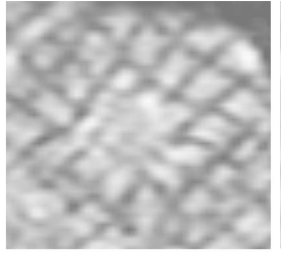

(d)

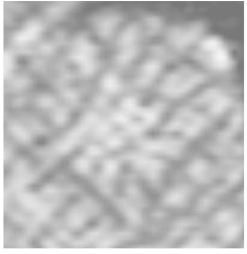

(e)

Fig. 8. MR tag (also known as Spatial Modulation of Magnetization (SPAMM)) images of one slice through a left ventricle at five points during systole. The tag grid is layed down in (a) at the end-diastole and the tags deform with the myocardical tissue from (b) through (e) four equally-spaced time points between ED and ES.. (Images courtesy of D. Metaxas, University of Pennsylvania).

based statistical difference measures that could quantitatively characterize lesion growth from MR images. While these efforts involve aspects of both segmentation and registration, the exploration of the time domain for deriving quantitative information is unique here. Further efforts in this area have begun to explore the utility of specific operators that could uncover useful information, such as the Jacobian of the deformation field between two registered image data sets [111]. In addition, it is important to note that time series analysis of mammographic data may ultimately be an imporant application area [112].

\subsubsection{Physics-Based Models}

In some medical image analysis application areas, the anatomical object or physiological process under study had been previously studied in vitro and, sometimes, in vivo by independent experimental techniques in an effort to develop forward models for simulation and/or analysis. Such modeling efforts, often found in the biomechanics and physiology literature, motivated a number of efforts aimed at recovering quantitative information from medical images by using these models as a basis for integrating imagederived information. Some of the initial work associated with physical modeling included ideas aimed at developing artificial forces related to the adherence of the model to features derived from the image data. These forces were then used as input to finite element models of Lagrangian mechanics, as noted above [51], and/or models capturing harmonic modes of temporal change [50]. In addition, as
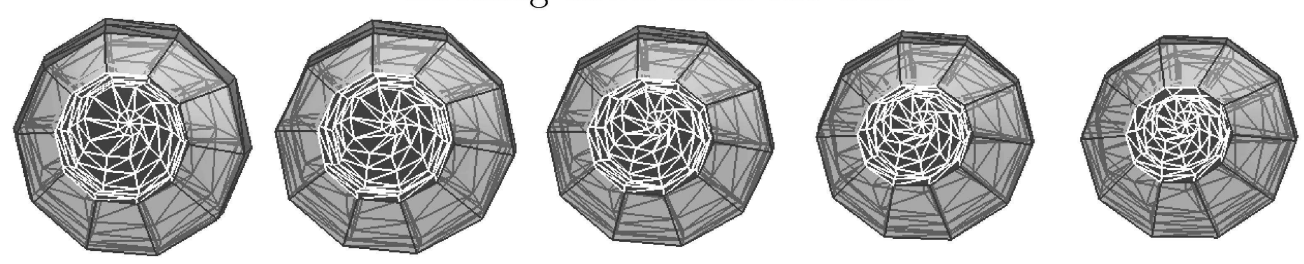

Looking from the side
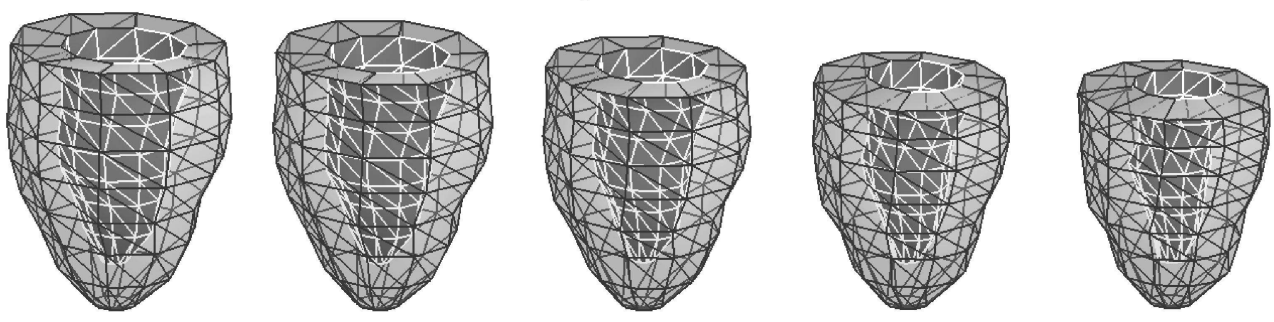

Looking at the apex

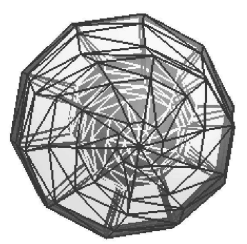

time $1(\mathrm{ED})$

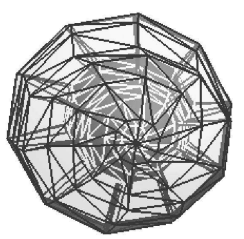

time 2

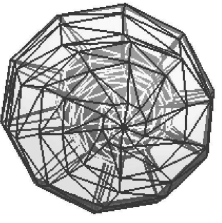

time 3
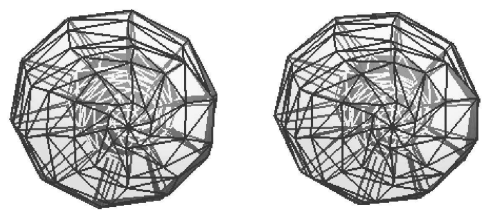

time 4

time $5(\mathrm{ES})$

Fig. 9. Fitted LV models during the same five time points during systole shown in Fig. 8, but using complete 3D data from two orthogonal MR tag acquitions. LV surfaces are formed using the methods described in [51]. (a) Looking down from the base. (b) Looking from the side. (c) Looking at the apex. (Figure courtesy of D. Metaxas, University of Pennsylvania). 
described above, biomechanical models have been used as the basis for developing image registration strategies, basically providing an interpolation paradigm [92]. In each of these cases, the models were not necessarily intended to capture true physical properties of the objects under study, but rather to provide a mathematical framework for smoothly integrating image-derived information and interpolating between missing data.

In the mid-to-later part of the 1990s, however, models based on actual physical properties were getting attention in a number of image analysis problems. Inspired by a wealth of effort in the cardiac biomechanics community (e.g., [113]), simple linear elastic models based on infinitesimal and then finite strain assumptions were used as the basis to integrate a set of displacements and velocities from MR image sequence data [107] in order to ultimately provide a dense set of left ventricular deformation information, useful for quantifying a variety of normal and abnormal states of the heart. Such models were also used to integrate MR tag-derived information from the right ventricle of the heart [114]. In another application gaining interest, using simple models based on crude measurements of brain deformation properties, researchers began to look at the problem of compensating for the brain shift that occurs between preoperative imaging and intraoperative neurosurgical procedures. Here, the challenge is to deform the densely acquired preoperative 3D anatomical image information (usually $\mathrm{MR}$ ) to a correct intraoperative position that can change from the preoperative state by as much as $1 \mathrm{~cm}$, using some form of intraoperative measurements. Carried along with the deformed anatomy are 3D maps of functional brain information derived from a variety of imaging techniques, including functional MRI (fMRI), and functional PET and SPECT. The models used to date in this application include ones based on dense solid mechanics [115], mass-spring networks [116], [139] and porous models based on soil mechanics [117]. A key challenge here is to understand what intraoperative data, image-based or otherwise, will really be available routinely. To date, possibilities range from recovering a sparse set of points [116] to using ultrasound data [118] to using more complete information from intraoperative MRI scanners [119]. Issues of accuracy, utility, and cost must all be carefully considered here in the long run to decide among the options.

As this decade draws to a close, a number of researchers have begun to look at a range of problems other than the heart and brain where physical modeling may play a key role for recovering and analyzing image-based information, including simulation and analysis of the liver, the gall bladder, and bony and soft tissue structure in and around the hands, face, and knees. Interestingly, the increased availability of high spatial and temporal resolution image data, especially of soft tissue structure through MRI and ultrasound, will continue to enhance the field's ability to obtain accurate model parameter information and, hence, make physical models a useful platform for more accurately integrating imagederived data. However, the effective use of these models will require new knowledge from a variety of areas, especially from complimentary fields such as biomechanics.

\subsection{New Application Areas: Toward Image-Guided Interaction During Intervention}

The ever-improving capabilities of medical image acquisition, computers, and computer graphics technology opened new horizons for medical image analysis researchers into areas of interactive analysis and, especially, image-guided intervention in the 1990s. As imaging and the interpretation of information from images become key parts of a surgeon's or interventionalist's procedure, the problems of identifying or segmenting structure from both preoperative and intraoperative information now must be addressed in near real-time. Furthermore, the registration of different types of intraoperative information to preoperative images (and image-based atlases) presents a variety of new challenges. One such problem was alluded to above in terms of brain shift during neurosurgery, where both modeling and intraoperative data gathering are ongoing difficult problems. One example of a state-of-the-art image analysis / visualization system being used in neurosurgery is illustrated by the work of a Harvard/MIT collaborative team, as shown in Fig. 11. Here, structured light is used as a basis to register intraoperative head position to preoperative structure and the image analysis efforts result in a visualization that is presented to the surgeon where the position of a tumor can be more clearly seen in relation to the rest of the head [120]. Another clinically successful example is the use of image guidance for hip replacement surgery. Important and interesting work in this area in terms of image analysis was performed by a team from Carnegie Mellon University and Shadyside Hospital in Pittsburgh [121], [122]. Here, surgical planning is performed from segmented CT images and the software platform includes a component that permits the surgeon to predict the range of motion that the patient can expect after surgery. It is important to note that both of these efforts employ primarily rigid registration to date, although many image-guided surgery and intervention problems require knowledge of, and compensation for, the nonrigid movement of soft tissue structure. While the ideas proposed above under physics-based modeling begin to address this, the need for real-time interaction puts more constraints on the types of processing strategies that can be used. In this regard, some interesting efforts were proposed during the mid-1990s to study volumetric models that can used in closer to real time, such as those described by Gibson et al. [123], but much more work needs to be done, as noted in recent overview of work in surgery simulation (see [9] and related articles in same issue).

Another key use of image analysis is in minimally invasive surgical procedures. One interesting example of is in the replacement of conventional vascular surgery by endovascular procedures for the treatment of abdominal aortic aneurysms [124] (see Fig. 10 (upper right)). In this procedure, a prosthesis is inserted into the abdominal aorta via the femoral arteries. Insertion is done using a delivery system which contains a folded prosthesis. If the prosthesis is at the right position in the aorta, it can be deployed. The whole procedure is performed under fluoroscopic control. At the University Medical Center Utrecht in The Netherlands, image analysis tools have been developed for preoperative planning and training using this procedure, 

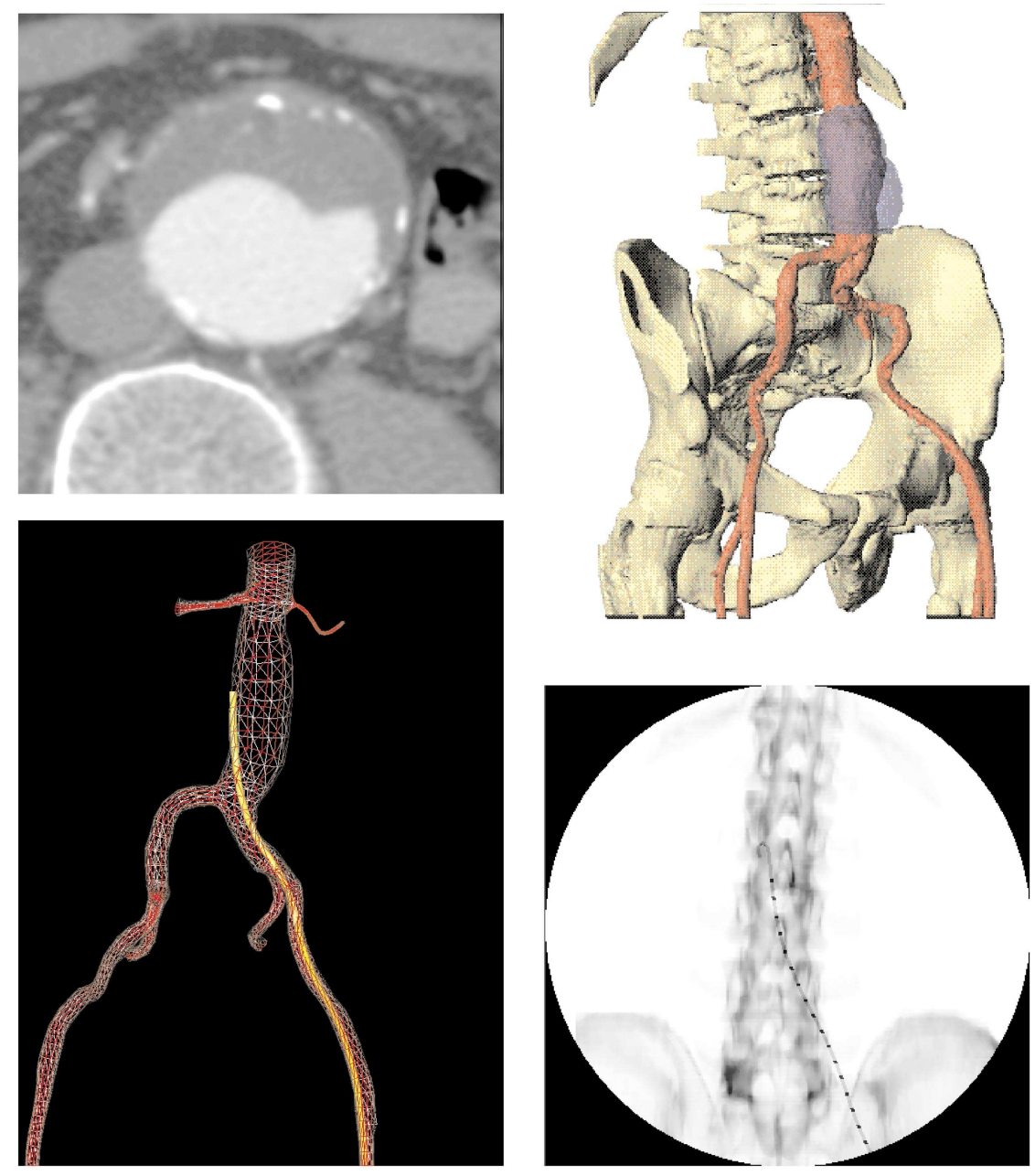

Fig. 10. Upper left: A single raw CT image showing a cross-sectional slice perpendicular to the lumen. Upper right: Surface rendering of an abdominal aortic aneursm, assembled from manual expert segmentation of a set of CT image slices, showning lumen and thrombus. Lower left: The segmentation of image data from the same patient is converted into an anatomical model of the vasculature for use in planning and simulation. Lower right: A simulated fluoroscopic image using a phantom dataset and a model similar to the one shown in the lower left of this figure [124], [125], [126], [127]. (Figure courtesy of Image Sciences Institute, University Medical Center Utrecht).

as shown in Fig. 10. Here, a typical CT Angiographic (CTA) image slice is shown in the upper left. The manual segmentation of a patient with a large aneurysm is then shown in the upper right. A semiautomated technique has been developed to replace the manual segmentation of the aorta, femoral artery, and iliac artery. This is done in two steps [125], [126] by first finding the central vessel axis and then locating the vessel wall. Once a segmentation is available, a model of the anatomy can be developed, as shown in the bottom left figure. One can also produce

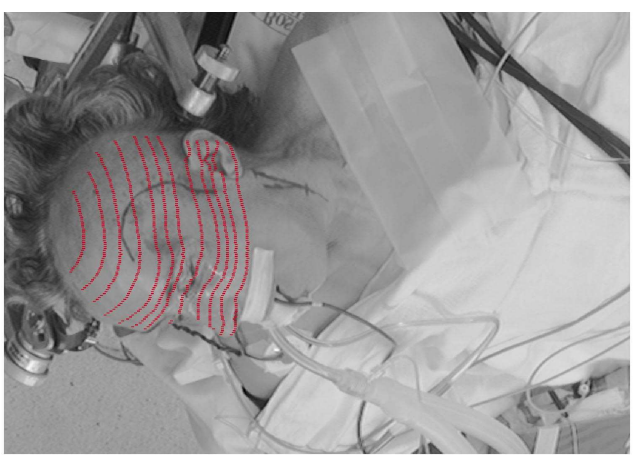

(a)

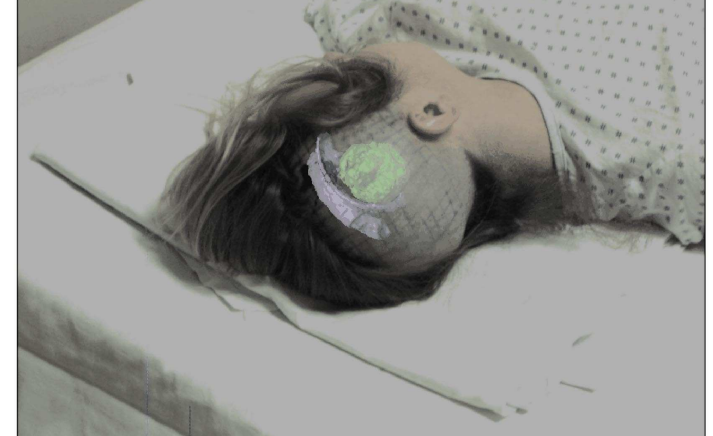

(b)

Fig. 11. Image-guided neurosurgery, as described in [120]. (a) Structured light patterns are overlayed on the patient and used to help register the patient to preoperative medical image information. (b) Virtual overlay of the ventricles and a tumor on the external patient frame. (Images provided by E. Grimson and M. Leventon, MIT). 
models of instruments that interact with this anatomical model [127] and can show the introductory trajectory of a prosthesis (e.g., as shown in yellow on the bottom left of the figure). Furthermore, simulated flouroscopic images can be produced by using these models (see Fig. 10 (lower right), in conjunction with the CTA image data of a phantom in order to provide a radiation-exposure-free environment for interventionalists to train in the use of this procedure.

Ultimately, complete, clinically useful, image-guided surgical and interventional systems must accurately, reproducibly, and interactively locate internal anatomical structure and display quantitative physiological function in relation to the surgeon's external reference field. In addition, the system must have the capability of relating the position of a variety of surgical and interventional instruments to the frame of reference. While some efforts have begun to address these issues (e.g., [128]), significant work remains to produce robust image-guided platforms. For an overview of the state of the art in this area through about 1996, the reader is referred to an excellent reference text edited by Taylor et al. [129].

\section{AND BeyOnd: Many Difficult Challenges Remain}

Medical image analysis in 1999 continues to be an active area of research, with many encouraging results, but also with a number of difficult problems still to be addressed. As a way of taking stock, perhaps the current state of the art of the field could be evaluated at this point in two ways: 1) what's currently in use in something close to routine clinical practice and 2) what promising ideas will be likely to affect clinical/biomedical science and clinical practice in the years to come. In the first category, as an example, a variety of commercial medical imaging equipment now comes loaded with simple forms of image processing and analysis algorithms. While these might range from basic thresholding to semiautomated forms of active contour algorithms, they do exist and radiologists and technologists use them to some extent. This is especially true in the nuclear medicine arena where regions of interest are routinely specified in order to quantify changes in radiopharmaceutical concentration. In addition, several stand-alone commercial image analysis platforms incorporating a range of medical image analysis developments now exist that are used by many medical researchers and, and to some extent, clinicians. Perhaps most prominent of these is the ANALYZE package developed at the Mayo Clinic [130]. Another is the MEDX package [131], used primarily to analyze functional MRI (fMRI) and functional nuclear medicine data. In addition, there has been a flurry of commercial software platform development activity in the areas of image-guided surgery (e.g., for neurosurgery [132]) and intervention (e.g., for radiotherapy [133]), incorporating basic registration schemes that mostly utilize external markers, but with the promise of incorporating more sophisticated algorithms. Furthermore, as noted earlier, the standard clinical equipment used in digital subtraction angiography (DSA) makes use of motion correction strategies originally developed within the medical image analysis community. In addition, a variety of 2D image analysis techniques are already strengthening clinical breast cancer diagnosis at a number of institutions [30].

In the short term, over the next decade or so, it is likely that more sophisticated forms of a range of algorithms, such as deformable surface segmentation, intensity-based segmentation, including bias field correction, informationtheoretic (i.e., mutual information-driven) and/or intensity-based image registration, and robust-point-based image registration will find their way into more and more software packages that are included as part of commercial medical imaging equipment and stand-alone medical image analysis workstations. They will be used in application areas ranging from diagnostic imaging to radiotherapy treatment planning to surgical navigation. As this occurs, it is important that the development of such platforms be closely integrated with the current thinking in the end-user communities in order to provide feedback.

\subsection{Key Challenges}

The research field of medical image analysis also has many difficult challenges ahead, both in terms of addressing the practical needs of its user community (a range of physicians and biomedical scientists) as well as developing theoretical substrates that would help put the field on firmer scientific and technological footing. First, the work in general must be developed and clearly motivated from the underlying biological problems that are being addressed. Many of these problems require the analysis of not only normal structure and function, but also of different abnormal, pathological, or disease states. To date, all too often image analysis algorithm development ignores the latter. In addition, the bridging of information uncovered at the macroscopic (perhaps "organ") level to information being uncovered at the molecular and cellular level needs to occur to a greater extent, and imaging and image analysis have an important role to play here in terms of analyzing data and integrating information. As genes are mapped and microstructure better characterized, opportunities may arise where quantitative image analysis of the organ-level images that we typically deal with may benefit from findings at the molecular and cellular levels. Similarly, findings at the organ level may be used in conjunction with microscopy to gain new insights into physiological function. Such lines of research may become more and more relevant here at the turn of the century as the "macro" and the "micro" begin to merge.

Second, it is felt that the processing and analysis strategies that are proposed must be put more in touch with image acquisition strategies. MRI research is both the most likely candidate for this, as well an example of where it has barely happened to date. Although some image analysis groups have made solid investments in the direction of integrating MR physics knowledge into image analysis strategies (e.g., [134], [135], [73]), in general the MR physics community and the medical image analysis community go their own separate ways in terms of research efforts, as well as in the choice of publication venues (conferences and journals). Interesting observations can even be made about the methodological tools each field focuses on at the moment: For instance, many $\mathrm{MR}$ 
researchers are particularly interested in characterizing brain function and are quite interested in the low level interactions of the NMR signal with biological tissue. However, surprisingly, many are willing to accept rather weak approaches to localizing the anatomical zones from which the activations occur, relying on rather crude piecewise atlases such as Talairach and registration approaches that primarily rely on a few manually delineated points. On the other hand, many medical image analysis researchers still do not fully appreciate the underlying MR physics that are the very basis of the data that they are working with when attempting to quantify structure and function. In general, concepts of task-driven design and feedback between the output of an analysis algorithm and the setting of image acquisition parameters are largely ignored. Neither group of researchers has fully analyzed the variability in the acquisition equipment, the analysis algorithms, and the interaction of human operators with both in order to characterize the complete information processing chain. The above issues are the case not only in MRI efforts, but in work related to other imaging modalities as well. There are, however, some groups making strong efforts to characterize the physics of the imaging process prior to analysis. Examples include work in mammography (e.g., [136]), ultrasound (e.g., [137]), and nuclear medicine (e.g., [138]).

Third, the field needs to think more seriously about defining a set of core principles that drive our efforts or, at least in retrospect, identifying the themes that run through the major research branches of our field, preferably in more analytical terms. So far, the bulk of the efforts over the last 20 years have been driven by assessing a host of separate, but somewhat related, medical and biomedical tasks and then applying engineering principles to design appropriate solutions without much consideration given to assessing what these solutions might have in common. The unique aspects of medical image analysis problems noted at the outset of this paper-the manner in which the data are acquired, the deformable nature of the objects involved, the nonrigidity of motion, the statistical variation of both normal and abnormal information-might be used to develop unifying principles that could help guide design methodology. Such efforts serve a variety of purposes, including helping the field to see itself more clearly so as to not be "reinventing the wheel" as often, and also allowing researchers to communicate more confidently about algorithm design both within the community and to potential user groups. Although this can all sound quite abstract, it is felt by the authors that this is more within our grasp than we realize. For instance, when dealing with multiple patient datasets or trying to combine information across several datasets, many, if not most, segmentation and registration strategies rely on primarily geometrical principles-i.e., at the end of the day, it is often geometry in one form or another that permits us to say that we've found a particular structure or that we've brought two datasets into alignment. This isn't to say that intensity-related information isn't a key part of the process for many tasks, just that geometry and geometrical analysis likely are the dominant common themes that run through most applications and tasks. The reader can look over recent review articles on segmentation and registration (e.g., [7], [8]) and assess this on her/his own as well. In another way, when trying to analyze information within image data from the same patient, we can begin to think of more physically-based models that can deform a structural or a functional map at one time into its realization at a new time point. Here, the use of models that represent deforming soft tissue that can be guided from data derived over time from a single patient's images clearly makes sense (e.g., [117], [139], [107] from above). Finally, we would note that many medical image analysis tasks are taken in isolation, rather than considered together. For instance, segmentation and registration are often just pieces of the same basic underlying problems of identifying structure and function in different, but related, spaces. Integrated decision making that better combines constraints from the individual processing tasks would help constrain the individual task goals and, in general, make the algorithms more robust.

A fourth major challenge for the field is one that remains a critical issue in terms of all practical and theoretical development: The need to develop appropriate validation and evaluation approaches. This challenge has a variety of aspects to it, some of which people in the field have been trying to address. First is the formation of common databases where algorithms can be compared and contrasted to each other. Our literature remains full of papers that evaluate algorithms on a few trial datasets from the home institution. This is sometimes in part due to simple lack of availability of a test set. As noted above, a very small set of test databases are beginning to be made available to the community. However, much more needs to be done in setting up such test databases, and funding agencies and review panels need to take this more seriously. The second issue in evaluation is the need to not only develop databases, but to cultivate research that will focus on the development of evaluation methodology. The one effort that has been heralded in recent years is the work of West and Fitzpatrick mentioned above [87], who were funded to both develop a database for image registration as well as the test strategy that would evaluate the strengths and weaknesses of rigid registration algorithms from all over the world. Although lauded by many members of the community as quite successful and useful, the emergence of follow-on programs has been nonexistent. Indeed, even this same group has encountered difficulty in trying to get follow-on efforts funded. We as a community cannot continue to cry out for better validation/evaluation and then not embrace such efforts more fully in our review panels and literature. In addition, efforts in the image quality assessment community that could be of great help in setting up evaluation strategies for image analysis remain largely separate from work in medical image analysis as defined in this paper, and this should be explored further.

Finally, it is felt that the medical image analysis community must become much more aware of the research activity in a host of other communities that surround us. Certainly, the modality-specific imaging physics communities and the research communities of a variety of clinical disciplines are examples of this. One current example is the 
surge of new research endeavors currently being carried out in the functional MRI area with relatively little input from the medical image analysis community. In addition, as noted above, we have largely ignored efforts that are going on in the biological sciences, ranging from the problems that are being addressed to the imaging technology that is being employed. Also, the explosion of medical image data being generated both clinically and from research laboratories provides opportunities for studying problems and developing systems related to indexing, searching, and sorting images based on pictorial information content, currently a topic of much interest in the computer vision community. Additionally, there is a variety of interest in developing surgical simulators that can directly make use of medicalimage-derived information. Attention to ideas such as those listed in this paragraph may provide new opportunities for specific research, but would also promote a broader awareness of such problems that could also (positively) influence current research trends in medical image analysis.

\subsection{Summary}

Medical image analysis will have a major role in biomedical research in the decades to come. In general, the field is flourishing, and this can be seen in a variety of ways, including the formation of new conferences and journals, as well as through the increased interest and activity from funding agencies. For instance, in the United States, a congressional bill has now been introduced to create a biomedical engineering institute at the National Institutes of Health (NIH), with imaging prominently named in a lead position and the analysis and processing of images given a lead role within that framework (see, for instance, http://www.nih.gov/ grants/becon/meeting99/index.htm). However, the challenges listed in the last section must be carefully addressed by researchers in the field if medical image analysis is to continue to flourish and be a key part of bioimaging science. It is felt that the community of scientists and engineers represented by the IEEE Transactions on Pattern Analysis and Machine Intelligence (PAMI) can play a significant role in the ongoing activity in the medical image analysis area, especially from the standpoints of developing integrated core information processing strategies and evaluation methodologies as mentioned in Section 5.1. In order to fulfill such a role, it is felt that the conferences organized under the PAMI rubric, and, ultimately, the journal itself, will have to embrace the idea that there are some interesting and fundamental algorithmic strategies that are coming out of the medical image analysis research area and that all work that utilizes medical images does not simply get categorized as a "medical applications" paper.

\section{ACKNOWLEDGMENTS}

The authors would like to thank Eric Grimson, Anond Rangarajan, Larry Staib, Hemant Tagare, and the Epidaure team at INRIA for their helpful discussions.

\section{REFERENCES}

[1] H.W. Strauss et al., "A Scintiphotographic Method for Measuring Left Ventricular Ejection Fraction in Man Without Cardiac Catheterization," Am. J. Cardiology, vol. 28, pp. 985991, Nov. 1971.

[2] J. Sklansky and D. Ballard, "Tumor Detection in Radiographs," Computers and Biomedical Research, vol. 6, no. 4, pp. 299-321, Aug. 1973.

[3] S. Pizer and A.E. Todd-Pokropek, "Improvement of Scintigrams by Computer Processing," Seminars in Nuclear Medicine, vol. 8, no. 2, pp. 125-146, Apr. 1978.

[4] "Forward," Proc. Information Processing on Medical Imaging, A. Kuba, M. Samal, and A. Todd-Pokropek, eds., pp. 1-508, 1999.

[5] G. Gerig, T. Pun, and O. Ratib, "Image Analysis and Computer Vision in Medicine," Computerized Medical Imaging and Graphics, vol. 18, no. 2, pp. 85-96, 1994.

[6] N. Ayache, "Medical Computer Vision, Virtual Reality and Robotics," Image and Vision Computing, vol. 13, no. 4, pp. 295313, 1995. also available at http://www. inria.fr/epidaure/ personnel/ayache/ayache.html.

[7] T. McInerney and D. Terzopolous, "Deformable Models in Medical Image Analysis: A Survey," Medical Image Analysis, vol. 1, no. 2, pp. 91-108, 1996.

[8] J.B.A. Maintz and M.A. Viergever, "A Survey of Medical Image Registration," Medical Image Analysis, vol. 2, no. 1, pp. 1-16, 1998.

[9] R. Shahidi, R. Tombropoulos, and R.P.A. Grzeszczuk, "Clinical Applications of Three-Dimensional Rendering of Medical Data Sets," Proc. IEEE, vol. 86, no. 3, pp. 555-568, Mar. 1998.

[10] S. Lavallee, "Registration for Computer Integrated Surgery: Methodology and State of the Art," Computer Integrated Surgery. R. Taylor, S. Lavallee, G. Burdea, and R. Mosges, eds., pp. 77-97, MIT Press, 1996.

[11] Proc. Int'l Symp. Medical Imaging and Interpretation (ISMII), pp. 1607, 1982.

[12] W. Schlegel, H. Scharfenberg, J. Doll, O. Pastyr, V. Sturm, G. Netzebend, and W. Lorenz, "CT Images as the Basis of Operation Planning in Stereotactic Neurosurgery," Proc. Int'l Symp. Medical Imaging and Interpretation (ISMII), pp. 172-177, 1982.

[13] M. Yachida, M. Ykeda, and S. Tsuji, "A Plan-Guided Analysis of Cineangiograms for Measurement of Dynamic Behavior of the Heart Wall," IEEE Trans. Pattern Analysis and Machine Intelligence, vol. 2, pp. 537-543, 1980.

[14] A. Martelli, "An Application of Heuristic Search Methods to Edge and Contour Detection," Comm. ACM, vol. 19, pp. 73-83, 1976.

[15] M. Yanagisawa, "Registration of Locally Distorted Images by Multiwindow Pattern Matching and Displacement Interpolation: The Proposal of an Algorithm and Its Application to Digital Subtraction Angiography," Proc. Seventh Int'l Conf. Pattern Recognition, M. Levine, ed., pp. 1,288-1,291, 1984.

[16] M. Fitzpatrick, J. Greffenstette, D. Pickens, M. Mazer, and J. Perry, "A System for Image Registration in Digital Subtraction Angiography," Information Processing in Medical Imaging. C. de Graaf and M. Viergever, eds., pp. 415-434, Plenum, 1987.

[17] E. Meijering, W. Niessen, and M. Viergever, "Retrospective Motion Correction in Digital Subtractive Angiography: A Review," IEEE Trans. Medical Imaging, vol. 18, no. 1, pp. 2-21, 1999.

[18] A. Klein, F. Lee, and A. Amini, "Quantitative Coronary Angiography with Deformable Spline Models," IEEE Trans. Medical Imaging, vol. 16, no. 5, pp. 468-482, 1997.

[19] H. Gelberg, B. Brundage, S. Glantz, and W. Parmley, "Quantitative Left Ventricular Wall Motion Analysis: A Comparison of Area, Chord and Radial Methods," Circulation, vol. 59, no. 5, pp. $991-1,000,1979$.

[20] M. Fujita, S. Sasayama, C. Kawai, S. Eiho, and M. Kuwahara, "Automatic Processing of Cineventriculograms for Analysis of Regional Myocardial Function," Circulation, vol. 63, no. 5, pp. 1,065-1,074, 1981.

[21] C.E. Metz, "Evaluation of Image Quality by ROC Analysis: Concepts, Techniques, and Future Possibilities," Japanese J. Radiological Technology, vol. 4, pp. 990-1,002, 1985.

[22] C.E. Metz, "ROC Methodology in Radiologic Imaging," Investigative Radiology, vol. 21, pp. 720-733, 1986.

[23] H. Barrett and C. Abbey, "Bayesian Detection of Random Signals on Random Backgrounds," Proc. Information Processing in Medical Imaging, J. Duncan and G. Gindi, eds., pp. 155-166, 1997.

[24] H. Barrett, K. Myers, and R. Wagner, "Beyond Signal Detection Theory," Proc. SPIE, vol. 626, pp. 231-239, 1986. 
[25] S. Zucker and R. Hummel, "A Three-Dimensional Edge Operator," IEEE Trans. Pattern Analysis and Machine Intelligence, vol. 3, no. 3, pp. 324-330, 1981.

[26] R. Schudy and D. Ballard, "Model-Detection of Cardiac Chambers in Ultrasound Images," Technical Report TR12, Computer Science Dept., Univ. of Rochester, Nov. 1978.

[27] R. Bajcsy, R. Lieberson, and M. Reivich, "A Computerized System for Elastic Matching of Deformed Radiographic Images to Idealized Atlas Images," J. Computer Assisted Tomography, vol. 7, pp. 618-625, 1983.

[28] H.P. Chan et al. "Improvements in Radiologists' Detection of Clustered Microcalcifications on Mammograms: The Potential of Computer-Aided Diagnosis," Investigative Radiology, vol. 25, pp. 1,102-1,110, 1990.

[29] H.P. Chan, K. Doi, S. Galhotra, C.J. Vyborny, H. MacMahon, and P.M. Jokich, "Image Feature Analysis and ComputerAided Diagnosis in Digital Radiography: Part 1 Automated Detection of Microcalcifications in Mammography," Medical Physics, vol. 14, pp. 538-548, 1987.

[30] F. Shtern, M. Vannier, and D. Winfield, "Report of the Working Group on Digital Mammography: Computer Aided Diagnosis and 3D Image Analysis and Display," Technical Report, U.S. Public Health Services's Office on Women's Health and the Nat'l Cancer Inst., Cambridge, Mass., Oct. 1998

[31] H. Niemann, H. Bunke, I. Hofmann, G. Sagerer, F. Wolf, and H. Feistel, "A Knowledge Based System for Analysis of Gated Blood Pool Studies," IEEE Trans. Pattern Analysis and Machine Intelligence, vol. 7, no. 3, pp. 246-259, 1985.

[32] J.S. Duncan, "Knowledge Directed Left Ventricular Boundary Detection in Equilibrium Radionuclide Angiocardiography," IEEE Trans. Medical Imaging, vol. 6, no. 4, pp. 325-336, 1987.

[33] C. Han, K. Lin, W. Wee, R. Mintz, and D. Purembka, "KnowledgeBased Image Analysis for Automated Boundary Extraction of Transesophageal Echocardiographic Left Ventricular Images," IEEE Trans. Medical Imaging, vol. 4, pp. 602-610, Dec. 1991.

[34] M. Kass, A. Witkin, and D. Terzopoulus, "Snakes: Active Contour Models," Int'l J. Computer Vision, vol. 1, pp. 312-331, 1988.

[35] M. O'Donnell, J. Gore, and W. Adams, "Towards an Automated Algorithm for NMR imaging II: Initial Segmentation Algorithm," Medical Physics, vol. 13, pp. 293-297, 1986.

[36] D. Ortendahl and J. Carlson, "Segmentation of Magnetic Resonance Images Using Fuzzy Clustering," Proc. Information Processing in Medical Imaging. C. de Graaf and M. Viergever, eds., pp. 91106, 1987.

[37] J. Koenderinck, "The Structure of Images," Biological Cybernetics, vol. 50, pp. 363-370, 1984.

[38] J. Gauch, W. Oliver, and S. Pizer, "Multiresolution Shape Descriptions and Their Applications in Medical Imaging," Proc. Information Processing in Medical Imaging. C. de Graaf and M. Viergever, eds., pp. 131-49, 1987.

[39] C. de Graaf, K. Vincken, M. Viergever, J. Koenderinck, F. Appelman, and O. Ying Lie, "A Hyperstack for the Segmentation of 3D Images," Proc. Information Processing in Medical Imaging, D.A. Ortendahl and J. Llacer, eds, pp. 399-413, 1989.

[40] D. Fritsch, S. Pizer, L. Yu, V. Johnson, and E. Chaney, "Segmentation of Medical Image Objects Using Deformable Shape Loci," Proc. Information Processing in Medical Imaging, J. Duncan and G. Gindi, eds., pp. 127-140, 1997.

[41] D. Morris, "An Evaluation of the Use of Texture Measures for Tissue Characterization of Ultraound Images of in Vivo Human Placenta," Ultrasound in Medicine and Biology, vol. 14, no. 1, pp. 387-395, 1988.

[42] J. Meunier, M. Bertrand, and G. Mailloux, "A Model for Dynamic Texture Analysis in Two-Dimensional Echocardiograms of the Myocardium," Proc. SPIE Pattern Recognition Acoustical Image, vol. 768, pp. 193-200, 1987.

[43] J. Meunier and M. Bertrand, "Ultrasonic Texture Motion Analysis: Theory and Simulation," IEEE Trans. Medical Imaging, vol. 14, no. 2, pp. 293-300, 1995.

[44] G. Jacob, J.A. Noble, M. Mulet-Parada, and A. Blake, "Evaluating a Robust Contour Tracker on Echocardiographic Sequences," Medical Image Analysis, vol. 3, no. 1, pp. 63-75, 1999.

[45] C.A. Pelizzari, G.T.Y. Chen, D.R. Spelbring, R.R. Weichselbaum, and C.-T. Chen, "Accurate Three-Dimensional Registration of CT, PET, and/or MR Images of the Brain," J. Computer Assisted Tomography, vol. 13, pp. 20-26, 1989.
[46] P.J. Besl and N.D. Mackay, "A Method for Registration of 3D Shapes," IEEE Trans. Pattern Analysis and Machine Intelligence, vol. 14, no. 2, pp. 239-256, Feb. 1992.

[47] J. Tsotsos, "Knowledge Organization and Its Role in Representation and Interpretation for Time-Varying Data: The ALVEN System," Computational Intelligence, vol. 1, no. 1, pp. 16-32, Feb. 1985.

[48] J. Duncan, R. Owen, L. Staib, and P. Anandan, "Measurement of Non-Rigid Motion Using Contour Shape Descriptors," Computer Vision and Pattern Recognition, G. Medioni and B. Horn, eds., pp. 318-324, IEEE CS Press, 1991.

[49] D. Metaxas and D. Terzopolous, "Constrained Deformable Superquadrics and Nonrigid Motion Tracking," Computer Vision and Pattern Recognition, G. Medioni and B. Horn, eds., pp. 337-343, IEEE CS Press, 1991.

[50] B. Horowitz and A. Pentland, "Recovery of Nonrigid Motion and Structure," Computer Vision and Pattern Recognition, G. Medioni and B. Horn, eds., pp. 325-330, IEEE CS Press, 1991.

[51] J. Park, D. Metaxas, and L. Axel, "Analysis of Left Ventricular Wall Motion Based on Volumetric Deformable Models and MRISPAMM," Medical Image Analysis, vol. 1, no. 1, pp. 53-71, 1996.

[52] N.C. Andreasen, G. Harris, T. Cizadlo, S. Arndt, and D.S. O'Leary, "Techniques for Measuring Sulcal/Gyral Patterns in the Brain as Visualized through Magnetic Resonance Scanning: BRAINPLOT," Proc. Nat'l Academy of Sciences USA, vol. 90, pp. 93-97, Jan. 1994.

[53] B.L. Holman, R.E. Zimmerman, K.A. Johnson, P.A. Carvalho, R.B. Schwartz, J.S. Loeffler, E. Alexander, C.A. Pelizzari, and G.T.Y. Chen, "Computer-Assisted Superimposition of Magnetic Resonance and High Resolution Technetium-99m-HMPAO and Thallium-201 SPECT Images of the Brain," J. Nuclear Medicine, vol. 32, pp. 1,478-1,484, 1991.

[54] P. Thompson, D. MacDonald, M. Mega, C. Holmes, A. Evans, and A. Toga, "Detection and Mapping of Abnormal Brain Structure with a Probabilistic Atlas of Cortical Surfaces," J. Computer Assisted Tomography, vol. 21, no. 4, pp. 567-581, July 1997.

[55] D. Dean, P. Buckley, F. Bookstein, J. Kamath, D. Kwon, L. Friedman, and C. Lys, "Three Dimensional MR-Based Morphometric Comparison of Schizophrenic and Normal Cerebral Ventricles," Visualization in Biomedical Computing, K.H. Hoehne and R. Kikinis, eds., pp. 363-372, Springer, 1996

[56] Proc. 1994 AAAI Spring Symp. Medical Applications of Computer Vision, W. Wells and E. Grimson, eds., pp. 1-200, Mar. 1994.

[57] T. Dohi, E. Grimson, and R. Kikinis, "Preface," Proc. Medical Image Computing and Computer Assisted Intervention (MICCAI), W. Wells, A. Colchester, and S. Delp, eds., pp. 1-1,256, Springer, 1998.

[58] L.D. Cohen and I. Cohen, "Finite Element Methods for Active Contour Models and Balloons for 2D and 3D Images," IEEE Trans. Pattern Analysis and Machine Intelligence, vol. 15, no. 11, pp. 1,1311,147, Nov. 1993.

[59] L.H. Staib and J.S. Duncan, "Model-Based Deformable Surface Finding for Medical Images," IEEE Trans. Medical Imaging, vol. 78, no. 5, pp. 720-731, Oct. 1996.

[60] T.F. Cootes, C.J. Taylor, D.H. Cooper, and J. Graham, "Active Shape Models-Their Training and Application," Computer Vision and Image Understanding, vol. 61, no. 1, pp. 38-59, 1995.

[61] G. Szekély, A. Kelemen, C. Brechbüler, and G. Gerig, "Segmentation of 3D Objects from MRI Volume Data Using Constrained Elastic Deformations of Flexible Fourier Surface Models," Computer Vision, Virtual Reality, and Robotics in Medicine. N. Ayache, ed., pp. 495-505, Berlin: Springer-Verlag, 1995.

[62] C. Xu and J. Prince, "A Generalized Gradient Vector Flow for Active Contour Models," Proc. Conf. Information Sciences and Systems, pp. 885-890, Mar. 1997.

[63] S. Osher and J. Sethian, "Fronts Propagation with Curvature Dependent Speed: Algorithms Based on Hamilton-Jacobi Formulations," J. Computational Physics, vol. 79, pp. 12-49, 1988.

[64] A. Yezzi, S. Kichenassamy, A. Kumar, P. Olver, and A. Tannenbaum, "A Geometric Snake Model for Segmentation of Medical Imagery," IEEE Trans. Medical Imaging, vol. 16, no. 2, pp. 199-209, 1997.

[65] P.C. Teo, G. Sapiro, and B.A. Wandell, "Creating Connected Representations of Cortical Gray Matter for Functional MRI Visualization," IEEE Trans. Medical Imaging, vol. 16, no. 6, pp. 852-863, 1997. 
[66] R. Malladi, J.A. Sethian, and B.C. Vemuri, "Shape Modeling with Front Propagation: A Level Set Approach," IEEE Trans. Pattern Analysis and Machine Intelligence, vol. 17, pp. 158-175, 1995.

[67] X. Zeng, L.H. Staib, R.T. Schultz, and J.S. Duncan, "Segmentation and Measurement of the Cortex from 3D MR Images," Medical Image Computing and Computer Assisted Intervention. pp. 519-530, Berlin: Springer-Verlag, 1998.

[68] L. Lorigo, O. Faugeras, W. Grimson, R. Keriven, R. Kikinis, and C. Westin, "Co-Dimension 2 Geodesic Active Contours for MRA Segmentation," Proc. Information Processing in Medical Imaging (IPMI), A. Kuba, M. Samal, and A. Todd-Pokropek, eds., pp. 126$139,1999$.

[69] S.C. Zhu, T.S. Lee, and A.L. Yuille, "Region Competition: Unifying Snakes, Region Growing and Bayes/MDL for Multi-Band Image Segmentation," Proc. Int'l Conf. Computer Vision, 1995.

[70] A. Chakraborty, L.H. Staib, and J.S. Duncan, "Deformable Boundary Finding in Medical Images by Integrating Gradient and Region Information," IEEE Trans. Medical Imaging, vol. 15, no. 6, pp. 859-870, Dec. 1996.

[71] T. Cootes, C. Beeston, G. Edwards, and C. Taylor, "A Unified Framework for Atlas Matching Using Active Appearance Models," Proc. Information Processing in Medical Imaging (IPMI99), pp. 322-333, Visegrad, Hungary, June 1999.

[72] W.M. Wells, W. Grimson, R. Kikinis, and F.A. Jolesz, "Statistical Intensity Correction and Segmentation of MRI Data," Proc. Visualization of Biomedical Computing 1994, SPIE 2359, R.A. Robb, ed., pp. 148-159, 1994.

[73] J. Sled, A. Zijdenbos, and A. Evans, "A Nonparametric Method for Automatic Correction of Intensity Nonhomogeneity in MRI Data," IEEE Trans. Medical Imaging, vol. 17, pp. 87-97, Feb. 1998.

[74] D. Pham and J. Prince, "An Adaptive Fuzzy Segmentation Algorithm for Three-Dimensional Magnetic Resonance Images," Information Processing in Medical Imaging. A. Kuba, M. Samal, and A. Todd-Pokropek, eds., pp. 140-153, 1999.

[75] D.L. Collins, A.P. Zijdenbos, V. Kollokian, J.G. Sled, N.J. Kabani, C.J. Holmes, and A.C. Evans, "Design and Construction of a Realistic Digital Brain Phantom," IEEE Trans. Medical Imaging, vol. 17, no. 3, pp. 463-468, June 1998.

[76] J.C. Rajapakse and F. Fruggel, "Segmentation of MR Images with Intensity Inhomogeneities," Image and Vision Computing, vol. 16, no. 3, pp. 165-180, Mar. 1998. http://neuro-www. mgh. harvard. $\mathrm{edu} / \mathrm{cma} / \mathrm{ibsr}$.

[77] H. Jiang, K. Holton, and R. Robb, "Image Registration of MultiModality 3-D Medical Images by Chamfer Matching," Proc. SPIE Biomedical Image Processing and Three-Dimensional Microscopy, vol. 1,660, pp. 356-366, 1992.

[78] R.A. Robb and D.P. Hanson, "A Software System for Interactive and Quantitative Visualization of Multidimensional Biomedical Images," Austalasian Physical and Eng. Sciences in Medicine, vol. 14, pp. 9-30, 1991.

[79] J. Feldmar and N. Ayache, "Rigid, Affine and Locally Affine Registration of Free-Form Surfaces," Int'l. J. Computer Vision, vol. 18, no. 2, pp. 99-119, May 1996.

[80] A. Rangarajan, H. Chui, E. Mjolsness, S. Pappu, L. Davachi, P. Goldman-Rakic, and J. Duncan., "A Robust Point Matching Algorithm for Autoradiograph Alignment," Medical Image Analysis, vol. 4, no. 1, pp. 379-398, 1997.

[81] J.-P. Thirion, "New Feature Points Based on Geometric Invariants for 3D Image Registration," Int'l J. Computer Vision, vol. 18, no. 2, pp. 121-137, May 1996.

[82] A. Guéziec, X. Pennec, and N. Ayache, "Medical Image Registration Using Geometric Hashing," IEEE Computational Science and Eng., special issue on geometric hashing, vol. 4, no. 4, pp. 29-41, Oct.-Dec. 1997.

[83] R.P. Woods, J.C. Mazziotta, and S.R. Cherry, "MRI-PET Registration with Automated Algorithm," J. Computer Assisted Tomography, vol. 17, no. 4, pp. 536-546, 1993.

[84] W. Wells III, P. Viola, H. Atsumi, S. Nakajima, and R. Kikinis, "Multi-Modal Volume Registration by Maximization of Mutual Information," Medical Image Analysis, vol. 1, no. 1, pp. 35-52, 1996.

[85] F. Maes, A. Collignon, D. Vandermeulen, G. Marchal, and P. Suetens, "Multi-Modality Image Registration by Maximization of Mutual Information," IEEE Trans. Medical Imaging, vol. 16, no. 2, pp. 187-198, 1997.
[86] C.R. Meyer, J.L. Boes, B. Kim, P.H. Bland, G.L. LeCarpentier, J.B. Fowlkes, M.A. Roubidoux, and P.L. Carson, "Semiautomatic Registration of Volumetric Ultrasound Scans," Ultrasound in Medicine and Biology, vol. 25, pp. 339-347, 1999.

[87] J. West et al. "Comparison and Evaluation of Retrospective Intermodality Registration Techniques," J. Computer Assisted Tomography, vol. 21, no. 4, pp. 554-566, July-Aug. 1997.

[88] A. Roche, G. Malandain, X. Pennec, and N. Ayache, "The Correlation Ratio as a New Similarity Measure for Multimodal Image Registration," Proc. Medical Image Computing and ComputerAssisted Intervention (MICCAI 1998), pp. 1,115-1,123, Cambridge, Mass., Oct. 1998.

[89] C.R. Meyer, J.L. Boes, B. Kim, P. Bland, K.R. Zasadny, P.V. Kison, K. Koral, K.A. Frey, and R.L. Wahl, "Demonstration of Accuracy and Clinical Versatility of Mutual Information for Automatic Multimodality Image Fusion Using Affine and Thin Plate Spline Warped Geometric Deformations," Medical Image Analysis, vol. 3, pp. 195-206, 1997.

[90] F.L. Bookstein, "Principal Warps: Thin-Plate Splines and the Decomposition of Deformations," IEEE Trans. Pattern Analysis and Machine Intelligence, vol. 11, no. 6, pp. 567-585, June 1989.

[91] F. Bookstein, "Shape and the Information in Medical Images: A Decade of the Morphometric Systhesis," Proc. Workshop Math. Methods in Medical Image Analysis, pp. 2-12, June 1996.

[92] G. Christensen, R. Rabbitt, M. Miller, S. Joshi, U. Grenander, T. Coogan, and D. van Essen, "Topological Properties of Smooth Anatomic Maps" Proc. Information Processing in Medical Imaging. Y. Bizais, C. Barillot, and R. Di Paola, eds., pp. 101-112, 1995.

[93] G.E. Christensen, M.I. Miller, U. Grenander, and M.W. Vannier, "Individualizing Neuroanatomical Atlases Using a Massively Parallel Computer," Computer, pp. 32-38, Jan. 1996.

[94] M. Vaillant and C. Davatzikos, "Finding Parametric Representations of the Cortical Sulci Using an Active Contour Mode,l" Medical Image Analysis, vol. 1, no. 4, pp. 295-315, 1997.

[95] B. Verdonck, R. Nijlunsing, J. Cheung, D. Wever, A. Veldhuizen, S. Devillers, and S. Makram-Ebeid, "Computer Assisted Quantitative Analysis of Deformities of Human Spine," Medical Image Computing and Computer Assisted Intervention. pp. 822-831, Berlin: Springer, 1998.

[96] H. Benali, I. Buvat, J. Anton, M. Pelegrini, M. DiPaola, J. Bittoun, Y. Burnod, and R. DiPaola, "Space-Time Statistical Model for Functional MR Image Sequences," Proc. Information Processing in Medical Imaging (IPMI), pp. 285-298, 1997.

[97] F. Kruggel and D. von Cramon, "Physiologically Oriented Models of Hemodynamic Response in Functional MRI," Proc. Information Processing in Medical Imaging," pp. 294-307, 1999.

[98] E. Zerhouni, D. Parish, W. Rogers, A. Yang, and E. Shapiro, "Human Heart Tagging with MR Imaging-A Method for Noninvasive Assessment of Myocardial Motion," Radiology, vol. 169 , pp. 59-63, 1988.

[99] L. Axel and L. Dougherty, "MR Imaging of Motion with Spatial Modulation of Magnetization," Radiology, vol. 171, pp. 841-845, 1989.

[100] W. Kerwin and J. Prince, "Cardiac Material Markers from Tagged MR Images," Medical Image Analysis, vol. 2, no. 4, pp. 339-354, 1998.

[101] A. Amini, Y. Chen, R. Curwen, V. Mani, and J. Sun, "Coupled BSnake Grids and Constrained Thin-Plate Splines for Analysis of 2D Tissue Deformations from Tagged MRI," IEEE Trans. Medical Imaging, vol. 17, pp. 344-356, 1998.

[102] S.N. Gupta and J.L. Prince, "On Variable Brightness Optical Flow for Tagged MRI," Information Processing in Medical Imaging, pp. 323-334, June 1995.

[103] N. Pelc, R. Herfkens, A. Shimakawa, and D. Enzmann, "Phase Contrast Cine Magnetic Resonance Imaging," Magnetic Resonance Quarterly, vol. 7, no. 4, pp. 229-254, 1991.

[104] R.T. Constable, K. Rath, A. Sinusas, and J. Gore, "Development and Evaluation of Tracking Algorithms for Cardiac Wall Motion Analysis Using Phase Contrast Velocity Imaging," Magnetic Resonance in Medicine, vol. 32, pp. 33-42, 1994.

[105] F. Meyer, R.T. Constable, A. Sinusas, and J. Duncan, "Tracking Myocardial Deformation Using Phase Contrast Velocity Fields: A Stochastic Approach," IEEE Trans. Medical Imaging, vol. 15, no. 4, pp. $453-465,1996$.

[106] C. Kambhamettu and D. Goldgof, "Curvature-Based Approach to Point Correspondence Recovery in Conformal Nonrigid Motion," CVGIP: Image Understanding, vol. 60, no. 1, pp. 26-43, July 1994. 
[107] P. Shi, G. Robinson, R. Constable, A. Sinusas, and J. Duncan, “A Model-Based, Integrated Approach to Track Myocardial Deformation Using Displacement and Velocity Constraints," Proc. Int'l Conf. Computer Vision, E. Grimson, ed., pp. 687-693, June 1995.

[108] S. Benayoun, C. Nastar, and N. Ayache, "Dense Non-Rigid Motion Estimation in Sequences of 3D Images Using Differential Constraints," Proc. Computer Vision, Virtual Reality, and Robotics in Medicine, pp. 309-318, 1995.

[109] H.D. Tagare, "Non-Rigid Curve Correspondence for Estimating Heart Motion," Proc. Information Processing in Medical Imaging, pp. 489-494, June 1997.

[110] G. Gerig, D. Welti, C. Guttmann, A. Colchester, and G. Szekely, "Exploring the Discrimination Power of the Time Domain for Segmentation and Characterization of Lesions in Serial MR Data," Proc. Medical Image Computing and Computer Assisted Intervention. pp. 469-479, 1998.

[111] D. Rey, G. Subsol, H. Delingette, and N. Ayache, "Automatic Detection and Segmentation of Evolving Processes in 3D Medical Images: Application to Multiple Sclerosis," Proc. Information Processing in Medical Imaging, IPMI 1999, A. Kuba, M. Sámal, and A. Todd-Pokropek, eds., pp. 154-167, June 1999.

[112] M. Sallam and K. Bowyer, "Registration and Difference Analysis of Corresponding Mammorgam Images," Medical Image Analysis, vol. 3, no. 2, pp. 103-118, 1999.

[113] A. McCulloch and J. Omens, "Non-Homogeneous Analysis of Three-Dimensional Transmural Finite Deformation in Canine Ventricular Myocardium," J. Biomechanics, vol. 24, no. 7, pp. 539$548,1991$.

[114] E. Haber, D. Metaxas, and L. Axel, "Motion Analysis of the Right Ventricle from MR Images," Proc. Medical Image Computing and Computer Assisted Intervention, pp. 177-188, 1998.

[115] S. Kyriacou and C. Davatzikos, "A Biomechanical Model of Soft Tissue Deformation, with Applications to Non-Rigid Registration of Brain Images with Tumor Pathology," Medical Image Computing and Computer Assisted Intervention. pp. 531-538, Berlin: Springer, 1998.

[116] O. Skrinjar, D. Spencer, and J. Duncan, "Brain Shift Modeling for Use in Neurosurgery," Proc. Medical Image Computing and Computer Assisted Intervention, W. Wells, A. Colchester, and S. Delp, eds., pp. 641-649, 1998.

[117] M. Miga, K. Paulsen, F. Kennedy, J. Hoopes, A. Hartov, and D. Roberts, "Initial in Vivo Analysis of 3D Heterogeneous Brain Computations for Model-Updated Image-Guided Neurosurgery," Proc. Medical Image Computing and Computer Assisted Intervention, W. Wells, A. Colchester, and S. Delp, eds., pp. 743-752, Springer, 1998.

[118] R. Bucholz, R. Yeh, J. Trobaugh, L. McDurmont, C. Sturm, C. Baumann, J. Henderson, A. Levy, and P. Kessman, "The Correction of Stereotactic Inaccuracy Caused by Brain Shift Using an Intraoperative Ultrasound Device," Proc. Computer Vision, Virtual Reality and Robotics in Medicine-Medical Robotics and Computer Assisted Surgery, J. Troccaz, E. Grimson, and R. Mosges, eds., pp. 459-466, Mar. 1997.

[119] P. Black, T. Moriarty, E. Alexander, P. Stieg, E. Woodard, L. Gleason, C. Martin, R. Kikinis, R. Schwartz, and F. Jolesz, "Development and Implementation of Intraoperative Magnetic Resonance Imaging and Its Neurosurgical Applications," Neurosurgery, vol. 41, no. 4, pp. 831-845, 1997.

[120] W. Grimson, G. Ettinger, S. White, T. Lozano-Perez, W. Wells, and R. Kikinis, "An Automatic Registration Method for Frameless Stereotaxy, Image-Guided Surgery and Enhanced Reality Visualization," IEEE Trans. Medical Imaging, vol. 15, no. 2, pp. 129-140, Apr. 1996.

[121] C. Nikou, B. Jaramaz, and A. DiGioia, "Range of Motion after Total Hip Arthroplasty," Proc. Medical Image Computing and Computer Assisted Intervention, pp. 700-709, 1998.

[122] C. Nikou, B. Jaramaz, A. DiGioia, M. Blackwell, M. Romesberg, and M. Green, "POP: Preoperative Planning and Simulation Software for Total Hip Replacement Surgery," Medical Image Computing and Computer Assisted Intervention. pp. 868-875, Springer, 1999.

[123] S. Gibson, J. Samosky, A. Mor, C. Fyock, E. Grimson, T. Kanade, R. Kikinis, H. Lauer, N. McKenzie, S. Nakajima, H. Ohkami, R. Osborne, and A. Sawada, "Simulating Arthroscopic Knee Surgery Using Volumetric Object Representations," Proc. Computer Vision, Virtual Reality and Robotics in Medicine-Medical Robotics and Computer Assisted Surgery, J. Troccaz, E. Grimson, and R. Mosges, eds., pp. 369-378, Mar. 1997.
[124] M. Breeuwer et al. "The EASI Project-Improving the Effectiveness and Quality of Image Guided Surgery," IEEE Trans. Information Technology in Biomedicine, vol. 2, no. 3, pp. 156-168, 1998.

[125] O. Wink, W.J. Niessen, and M.A. Viergever, "Fast Quantification of Abdominal Aortic Aneurysms from CTA Volumes," Proc. Medical Image Computing and Computer Assisted Intervention, pp. 138-145, 1998.

[126] O. Wink, W.J. Niessen, J.J. Wever, I.A.J.M. Broeders, J.D. Blankensteijn, and M.A. Viergever, "Semi-Automated Quantification and Segmentation of Abdominal Aorta Aneurysms from CTA Volumes," Proc. Computer Assisted Radiology and Surgery (CARS 1999), pp. 208-212, 1999.

[127] T. van Walsum and M.A. Viergever, "Deformable B-Splines for Catheter Simulation," Proc. Computer Assisted Radiology and Surgery (CARS 1999), pp. 999, 1999.

[128] A. Colchester, J. Zhao, K. Holton-Tainter, C. Henri, N. Maitland, P. Roberts, C. Harris, and R. Evans., "Development and Preliminary Evaluation of VISLAN, A Surgical Planning and Guidance System Using Intra-Operative Video Imaging," Medical Image Analysis, vol. 1, no. 1, pp. 73-90, 1996.

[129] Computer Integrated Surgery, R. Taylor, S. Lavalle, G. Burdea, and R. Moesges, eds. MIT Press, 1996.

[130] R.A. Robb and D.P. Hanson, "ANALYZE: A Software System for Biomedical Image Analysis," Proc First Conf. Visualization Biomedical Computing, pp. 507-518, 1990.

[131] Sensor Systems, MEDx software package, Sterling, Va., 1999. http://www.sensor.com.

[132] Sofamor Danek, stealth station, Memphis, Tenn., 1999. http:// www. sofamordanek.com.

[133] NOMOS Corporation, PEACOCK software product, Sewickley, Pa., 1999. http://www.nomos.com.

[134] D. Atkinson, D. Hill, P. Stoyle, P. Summers, and S. Keevil, "Automatic Correction of Motion Artifacts in Magnetic Resonance Images Using an Entropy Focus Criterion," IEEE Trans. Medical Imaging, vol. 16, no. 6, pp. 903-918, 1997.

[135] C. Meyer, P. Bland, and J. Pipe, "Retrospective Correction of Intensity Inhomogeneities in MRI," IEEE Trans. Medical Imaging, vol. 14, pp. 36-41, 1997.

[136] R. Highnam, M. Brady, and B. Shepstone, "A Representation for Mammographic Image Processing," Medical Image Analysis, vol. 1, no. 1, pp. 1-18, 1996.

[137] M. Insana and T. Hall, "Parametric Ultrasound Imaging from Backscatter Coefficient Measurements: Image Formation and Interpretation," Ultrasonic Imaging, vol. 12, pp. 245-267, 1990.

[138] B. Tsui, E. Frey, X. Zhao, D. Lalush, R. Johnson, and W. McCartney, "The Importance and Implementation of Accurate 3D Compensation Methods for Quantitative Spect," Physics in Medicine and Biology, vol. 39, pp. 509-530, 1994.

[139] P. Edwards, D. Hill, J. Little, and D. Hawkes, "Deformation for Image Guided Intervention Using a Three Component Tissue Model," Proc. Information Processing in Medical Imaging, J. Duncan and G. Gindi, eds., pp. 218-231, 1997. 


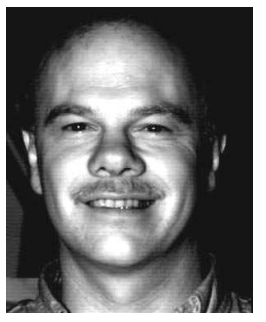

James S. Duncan received the BSEE degree from Lafayette College in Easton, Pennsylvania, in 1972, the MS degree in engineering from the University of California, Los Angeles, in 1975, and the $\mathrm{PhD}$ degree in electrical engineering from the University of Southern California, Los Angeles, in 1982. In 1973, he joined the staff of Hughes Aircraft Company, Electro-Optical and Data Systems Group, and participated in research and development projects related to signal and image processing for forward looking infrared (FLIR) imaging systems until 1983. During this time he held Hughes' masters, engineer, and doctoral fellowships. In 1983, he joined the faculty of Yale University, New Haven, Connecticut, where he currently is a professor of diagnostic radiology and electrical engineering. His research and teaching efforts have been in the areas of computer vision, image processing, and medical imaging. Currently, he is the director of undergraduate studies for the Yale program in biomedical engineering. $\mathrm{He}$ is also the director of the image processing and analysis group within diagnostic radiology. His current, specific research interests include the segmentation of deformable objects from images, the tracking of nonrigid object motion, and the integration of processing modules in vision systems, all with a special interest in using these approaches for medical image analysis.

Dr. Duncan was a Fulbright Research Scholar at the Universities of Amsterdam and Utrecht in the Netherlands during part of the 1993-1994 academic year. He is a member of Eta Kappa Nu and Sigma $\mathrm{Xi}$, is on the editorial board of the Journal of Mathematical Imaging and Vision, is an associate editor for the IEEE Transactions on Medical Imaging, and is a coeditor-in-chief of the journal Medical Image Analysis. In 1997, he chaired the 15th international meeting on Information Processing in Medical Imaging. In 1999, he was elected to be a fellow of the American Institute for Medical and Biological Engineering (AIMBE). $\mathrm{He}$ is currently serving as a member of the diagnostic imaging study section at the National Institute of Health $(\mathrm{NIH})$. Dr. Duncan is a senior member of the IEEE.

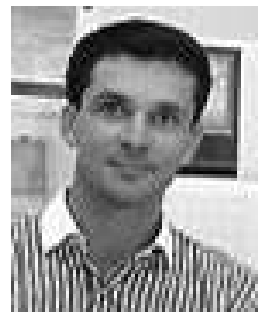

Nicholas Ayache received the $\mathrm{PhD}$ degree in 1983 and the Thèse d'Etat degree in 1988, both in computer science, from the University of Paris $\mathrm{XI}$, on topics related to model-based object recognition, passive stereovision, and multisensor fusion. His current research interests are in medical image processing and analysis (including shape and motion representation, rigid and nonrigid registration, tracking and analysis of deformable objects), simulation of surgery (including modeling of soft tissue), and image guided therapy (in particular, in the context of medical robotics). He is also involved in the analysis of functional images and their applications to medicine and neurosciences. Dr. Ayache is a research director at the French Research Institute on Computer Science and Automatic Control (INRIA), Sophia-Antipolis, France, where he has led the EPIDAURE research group in medical image analysis and robotics since 1992. He is also teaching graduate courses on computer vision at the Univiversity of Paris XI and Ecole Centrale and is consulting for a number of private companies, currently including Matra and Focus Imaging.

Dr. Ayache is currently coeditor-in-chief of the Journal of Medical Image Analysis, a member of the editorial board of the International Journal of Computer Vision and Computer Assisted Surgery, associate editor of IEEE Transactions on Medical Imaging, advisory editor of Videre-Computer Vision Research Journal, and Medical Imaging Technology (Japanese Society of Medical Imaging). He also served on the editorial board of the IEEE Transactions on Robotics and Automation (1990-1993) and as an area editor of the Journal of Computer Vision and Image Understanding (1994-1997).

Dr. Ayache is the author of the books Artificial Vision for Mobile Robots and Vision stéréoscopique et perception multisensorielle (InterEditions). He chaired the first International Conference on Computer Vision, Virtual Reality, and Robotics in Medicine (CVRMed) held in Nice in April 1995 and serves on the editorial boards of a number of conferences on medical imaging, computer vision, visualization and robotics including CVPR, ECCV, ICCV, and MICCAI. Dr. Ayache is a member of the IEEE. 